\title{
Improve the Performance of Advice Systems Based on Cooperative Liquidation Using Trust Relationships
}

\author{
EEva Diab Hriekes \\ Informatics Engineering Faculty - Albaath \\ University - Syria \\ evahriekes@gmail.com
}

\author{
Yosser AlSayed Souleiman AlAtassi \\ Informatics Engineering Faculty - \\ Albaath University - Syria \\ yalatasi@gmail.com
}

\begin{tabular}{l}
\hline ARTICLE INFO \\
\hline Submission date: $1 / 11 / 2018$ \\
Acceptance date: $14 / 11 / 201$ \\
Publication date: $20 / 2 / 2019$
\end{tabular}

ARTICLE INFO

Acceptance date: $14 / 11 / 20$

Publication date: $20 / 2 / 2019$

\begin{abstract}
Recommender systems are one of the recent inventions to deal with information overload problem and provide users with personalized recommendations that may be of their interests. Collaborative filtering is the most popular and widely used technique to build recommender systems and has been successfully employed in many applications. However, collaborative filtering suffers from several inherent issues that affect the recommendation accuracy such as: data sparsity and cold start problems caused by the lack of user ratings, so the recommendation results are often unsatisfactory. To address these problems, we propose a recommendation method called "MFGLT" that enhance the recommendation accuracy of collaborative filtering method using trust-based social networks by leveraging different user's situations (as a trustor and as a trustee) in these networks to model user preferences. Specifically, we propose model-based method that uses matrix factorization technique and exploit both local social context represented by modeling explicit user interactions and implicit user interactions with other users, and also the global social context represented by the user reputation in the whole social network for making recommendations. Experimental results based on real-world dataset demonstrate that our approach gives better performance than the other trust-aware recommendation approaches, in terms of prediction accuracy.
\end{abstract}

Keywords: Collaborative Filtering, Recommender Systems, Social Recommender Systems, Trust.

(C) Journal of University of Babylon for Pure and Applied Sciences (JUBES) by University of Babylon is licensed under a Creative Commons Attribution 4.0 International License 


\section{تحسين أداء النظم الناصحة المهتمدة علد التصفية التهاونيّة باستخدام علاقات \\ الثّقة}

\author{
يسر السيد سليمان الأتاسي \\ كلية الهندسة المعلوماتية - جامعة البعث - سوريا لانيانيان \\ yalatasi@gmail.com
}

\author{
ايفا دياب حريقص \\ كلية الهندسة المعلوماتية - جامعة البعث - سورياب \\ evahriekes@gmail.com
}

الخلاصة

إنّ النظم الناصحة هي أحد الابتكارات الحديثة للتعامل مع مشكلة الحمل الزائد للمعلومات وتزويد المستخدمين بتوصيات ذات طابع

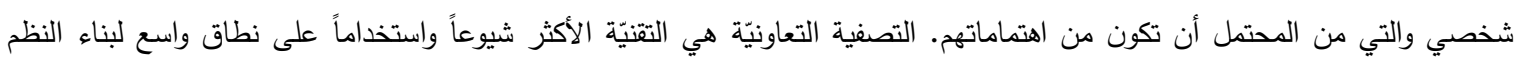

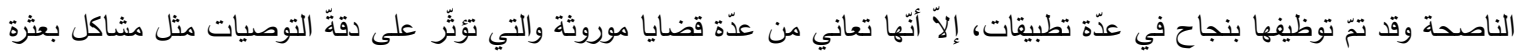

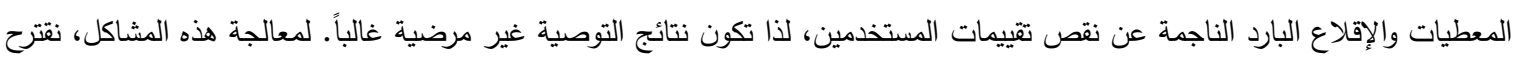

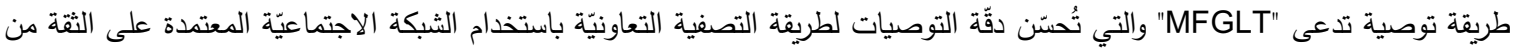
خلال الاستفادة من حالات المستخدم المختلفة (كواثق وكموثوق به) في هذه الشبكات لندذجة تفضيلات المستخدمين. على وجه التحديد،

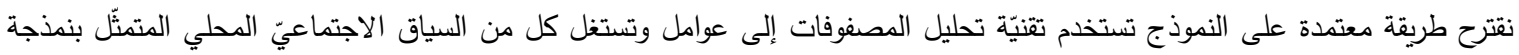

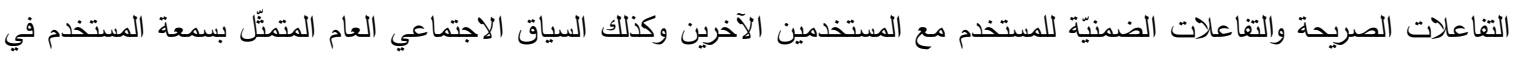

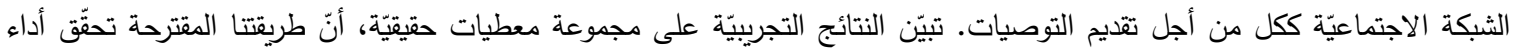
أفضل من الطرق الأخرى المعتدة على الثّقَة من حيث الدقّة التبوَّية. الكلمات الدّالة: التصفية التعاونيّة، النظم الناصحة، النظم الناصحة الاجتماعيّة، الثّقة

1 1

مع الكميّة المتزايدة بسرعة من المعلومات المتاحة على الإنترنت، ومع تطوّر تطبيقات الويب 2.0 التي غيّرت

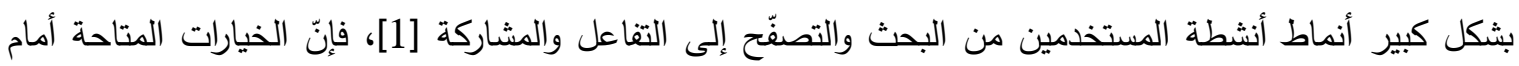
المستخدمين تتمو بشكل كبير، وهذا مائعرف بمشكلة الحمل الزائد للمعلومات، لذلك يصبح من الضروري أن يكون

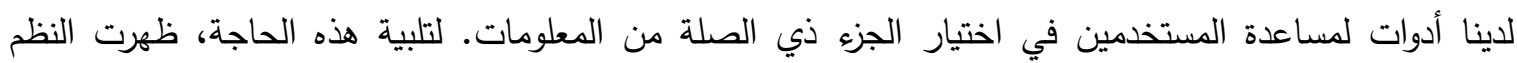
الناصحة، والتي هي تقنيّات وأدوات برمجيّة تزوّد باقتراحات وتوصيات ذات ذات طابع شخصي من أجل العناصر لتكون

ذات فائدة للمستخدم [2].

على الرغم من أنّه تمّ اقتراح عدّة أنواع من الطرق لبناء النظم الناصحة، فإنّ طريقة التصفية التعاونيّة [2] هي

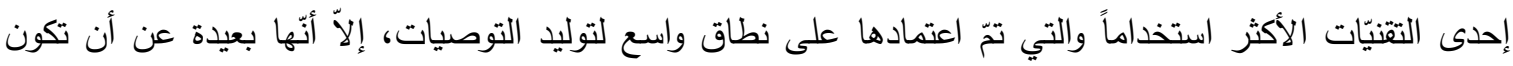

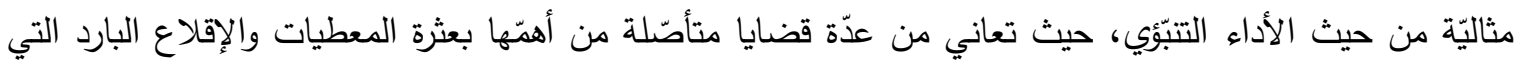
تؤثر على دقّة النظام الناصح [2]. لحلّ هذه القضايا ونمذجة تفضلات المستخدمين بشكل أكثر دقّة، يمكن دمج معلومات إضافيّة إلى طريقة التصفية التعاونيّة للتعويض عن معلومات التقييم الغير كافية مثل معلومات الثبكات

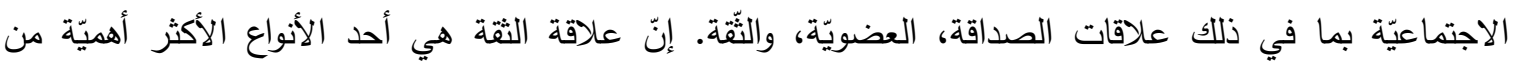


العلاقات الاجتماعيّة، نظراً لارتباطها القوي والإيجابي مع التشابه بين المستخدمين، وقد أظهرت العديد من الدراسات

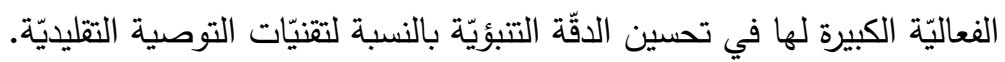
في الآونة الأخيرة، حصلت طرق التصفية التعاونيّة المعتمدة على النموذج، لاسيّما نماذج تحليل المصفوفات

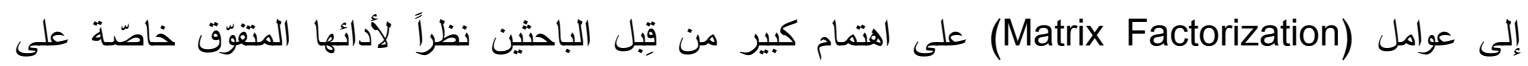

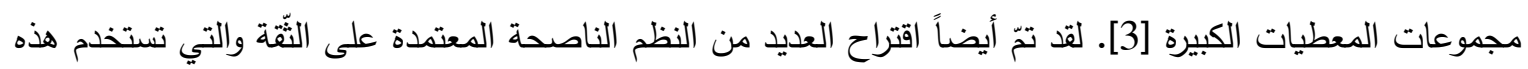

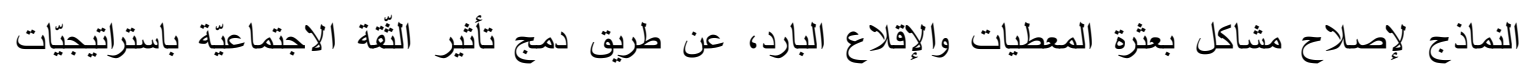

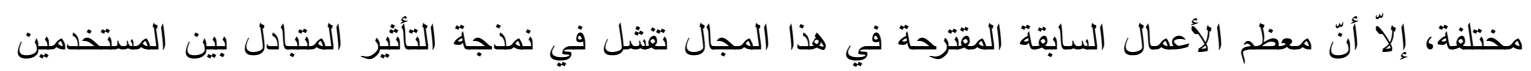

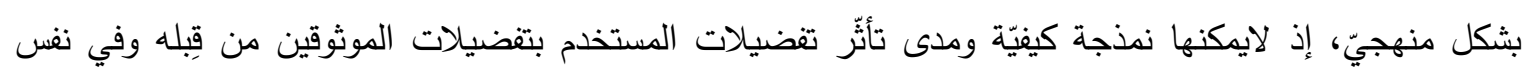
الوقت كيفيّة ومدى تأثير نفس المستخدم بالواثقين به، حيث تفضيلات المستخدمين كواثقين وتفضيلاتهم كموثوقين

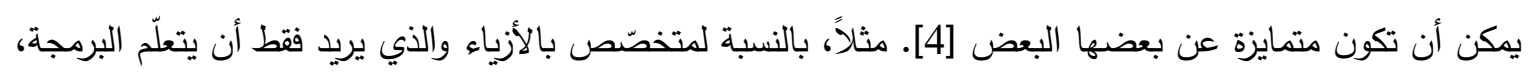
من المحتمل أكثر أن يثق بالكثير من المبرمجين في حين أنّه يكون موثوق من قِبِل العديد من المهتمين بالأزياء

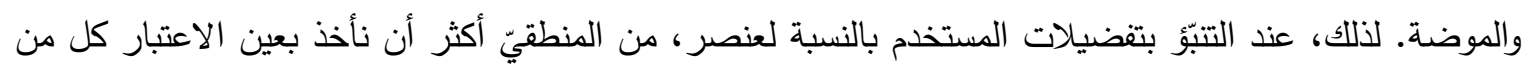

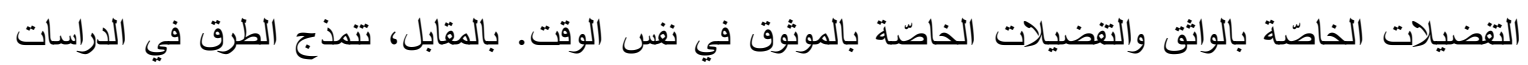

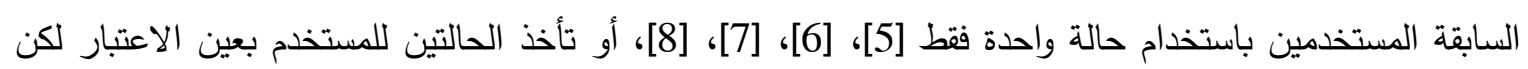
بشكل منفصل [4]. أي أنّه لايوجد أي تمييز بين الحالات المختلفة للمستخدم (كواثق وموثوق به) في عملية توليد التقييم.

علاوةً على ذللك، تعتمد معظم الطرق الموجودة على روابط الثّقة الصريحة بين المستخدمين، والتي على أساسها

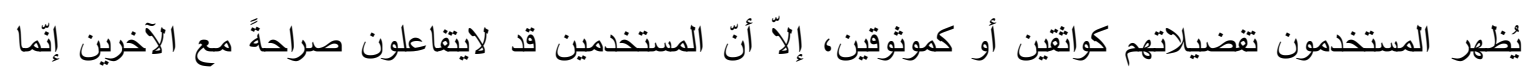

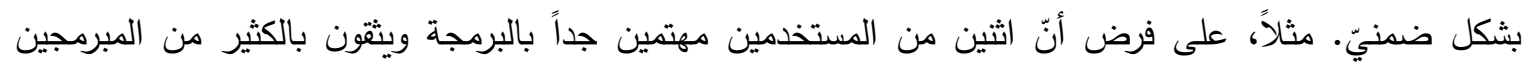

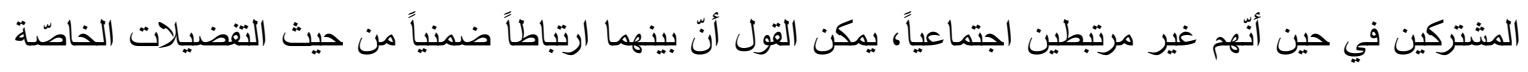

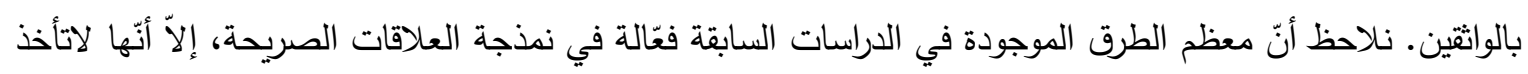

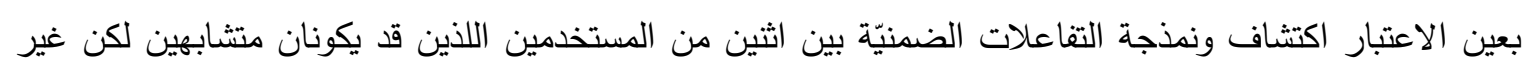
مرتبطين في شبكة الثّّة.

بالإضافة لذلك، يمكن رؤية العلاقات الاجتماعيّة بين المستخدمين من وجهة نظر محليّة ووجهة نظر عامّة [9]، الأولى تكثف العلاقات بين المستخدم وجيرانه بينما تكثف الثانية سمعة المستخدم في الشبكة الاجتماعيّة ككل.

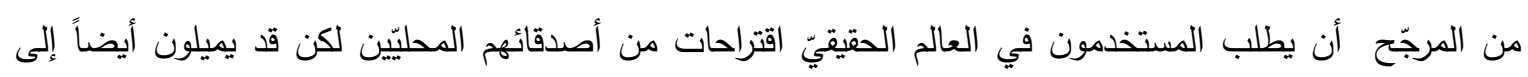

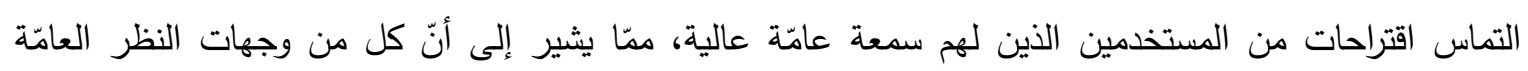

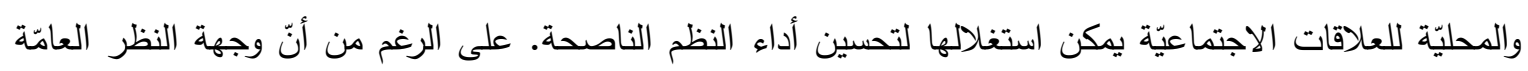

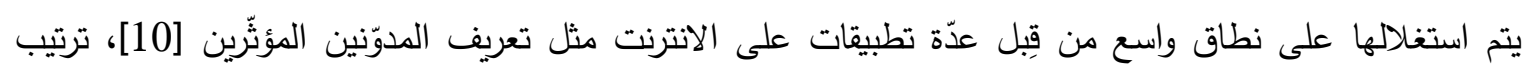

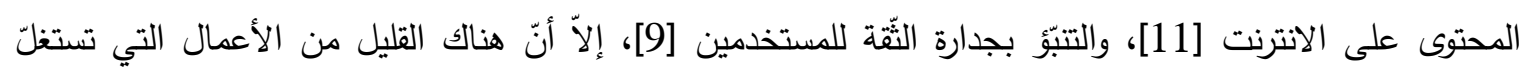
السياق الاجتماعيّ العام من أجل التوصيات.

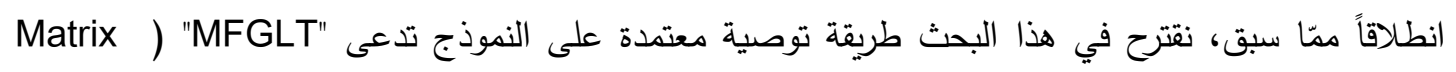
Factorization with Global Local Trust الثقة في نفس الوقت من أجل التوصيات. يقدّم هذا العمل استراتيجيّة جديدة لصهر معطيات التقييم ومعطيات الثّّة 
عن طريق الاستكشاف الكافي لكيفيّة توليد التقييمات المعروفة في ظل تأثير سلوكيّات الثّقة للمستخدمين والتي هي متشابكة عبر شبكة الثّّة الخاصّة بهم، بدلاً من مجرد تركيب نوعين من المعطيات ببساطة كما تفعل معظم الدراسات السابقة، وذلك للتعبير عن التأثير المتبادل للمستخدمين على آراء بعضهم بشكل منطقي أكثر ـ تستخدم الطريقة المقترحة تقنيّة تحليل المصفوفات لنمذجة تفضيلات المستخدمين بالنسبة للتوصيات المعتمدة على الثّّة، ويتم تعلّم تفضيلات الحالتين المختلفتين للمستخدمين عن طريق ندذجة التفاعلات الصريحة والضمنيّة بينهم. على وجه التحديد،

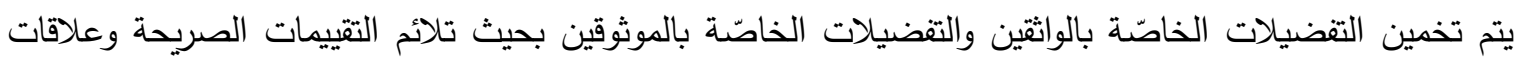

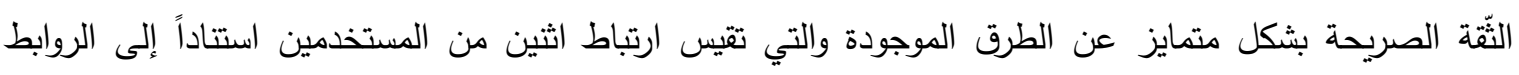

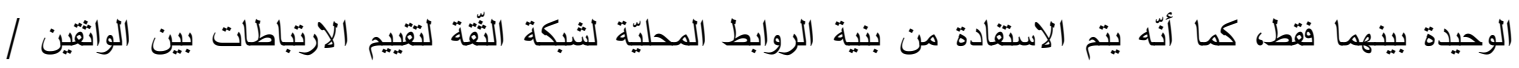

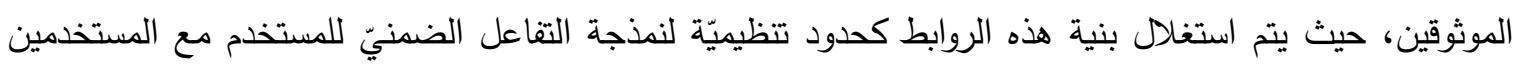

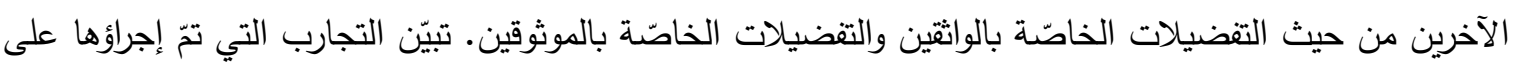

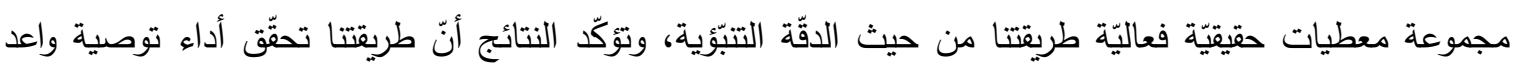
لاسيّما أنهّا فعّالة بالنسبة للمستخدمين ذوي الإقلاع البارد ويمكنها الاستفادة من معطيات الثّّة المبعثرة بشكل أكثر فعاليّة مقارنةً مع نظرائها الآخرين.

2. دراسات ذات صلة

إنّ دمج علاقات الثّّة مع طرق التصفية التعاونيّة المعتمدة على النموذج لتحسين دقّة التوصيات قد أصبح

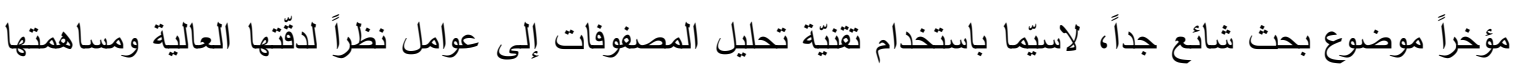

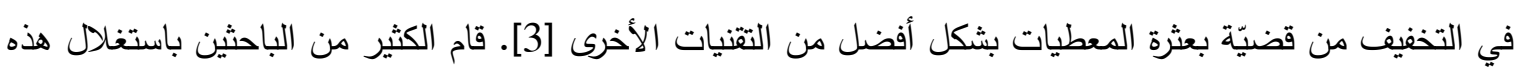

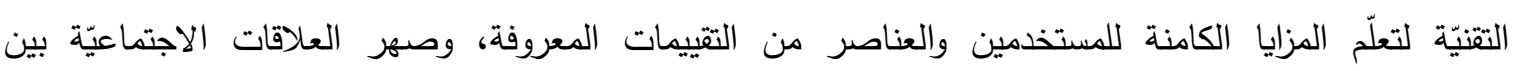

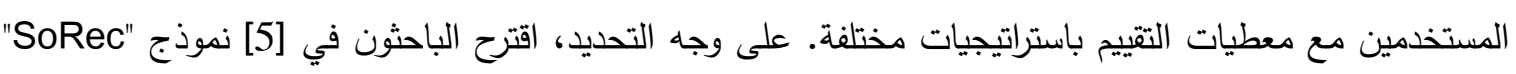
والذي يدمج بيان الثبكة الاجتماعيّة إلى نموذج تحليل المصفوفات الاحتمالي عن طريق تحليل مصفوفة التقييم

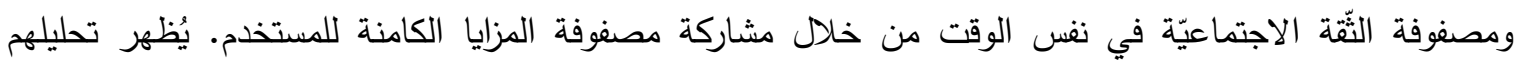
التجريبيّ أنّ طريقتهم تتفوق على نموذج تحليل المصفوفات الأساسيّ وعلى الطرق الأخرى المعتمدة على الذاكرة والتي فئي

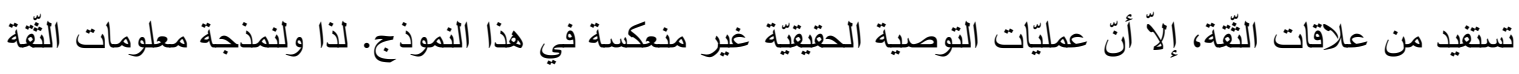
بشكل أكثر واقعيّة، اقترح نفس الباحثين في [6] نموذج "RSTE" والذي يفسّر قرار المستخدم بالتقييم كالتوازن بين ذوقه الخاص وأذواق جيرانه الموثوقين. تبيّن تجاربهم أنّ نموذجهم يتفوّق على طريقة تحليل المصفوفات الأساسيّة

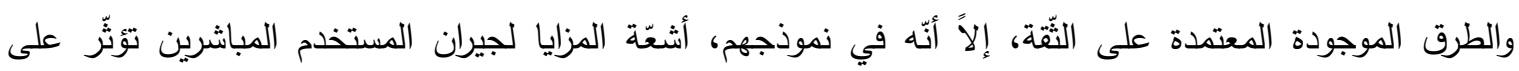

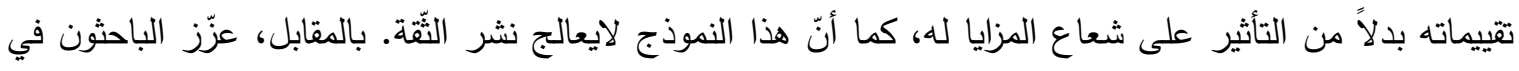

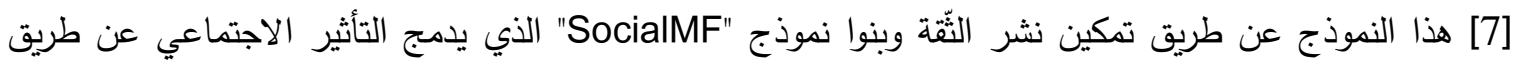
جعل المزايا الكامنة لكل مستخدم معتمدة على المزايا الكامنة لجيرانه المباشرين في الثبكة الاجتماعيّة. كذللك

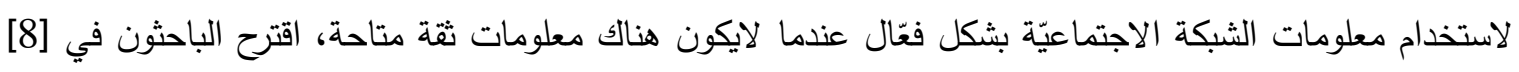
نموذج "SoReg" الذي يستخدم تقنيّة تحليل المصفوفات مع حد تتظيم اجتماعي يمثّل القيود الاجتماعيّة على النظم فئل

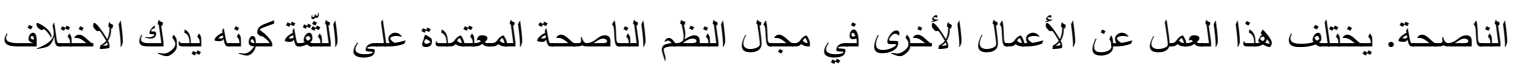
بين علاقة الثّقة وعلاقة الصداقة، كما أنّه يجبر تفضيلات المستخدم أن تكون أقرب إلى تفضيلات أصدقاءه في الشبكة الاجتماعيّة. 
تمّ أيضاً اقتراح نموذج "LOCABAL" في [12] والذي فيه اعتبر الباحثون كل من الثّّة العامّة والثّّة المحليّة كالمعلومات السياقيّة في نموذجهم، إنّه معتمد على نظريّات الارتباط الاجتماعي، وقد بيّنت نتائجهم أنّ استخدام الثّقة

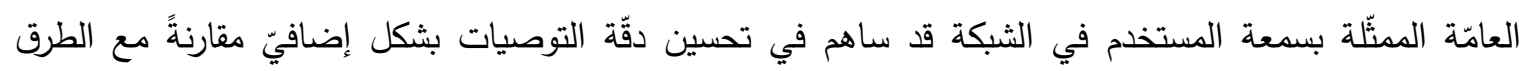

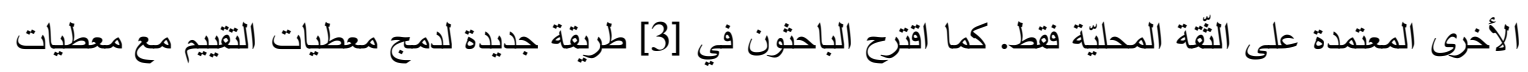

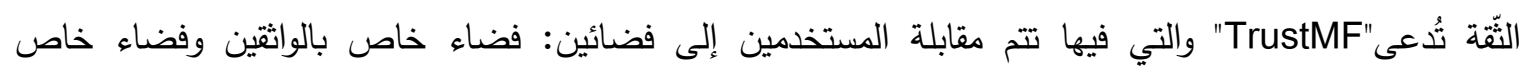
بالموثوقين، وتتم نمذجة التأثيرات من المستخدمين على تقييمات العناصر في كلا الفضائين. بيّنت تجاربهم أنّ هذاء النموذج يحقّق دقّة تتبؤيّة أفضل مقارنةً مع النماذج الأخرى المعتمدة على الثّة.

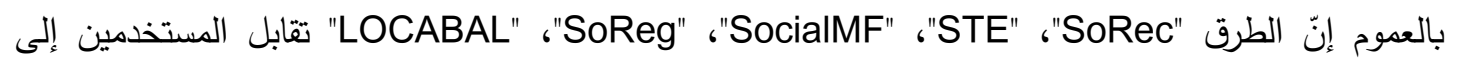
فضاء مزايا واحد، مهملةً حالات المستخدمين المختلفة، بينما العمل الأكثر صلة بعملنا وهو "TrustMF"، يتم فيه لفيه

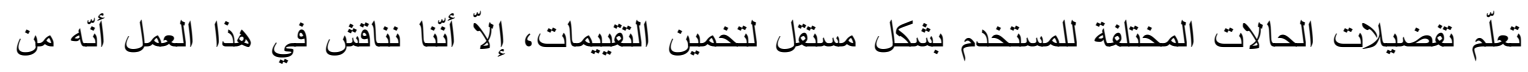

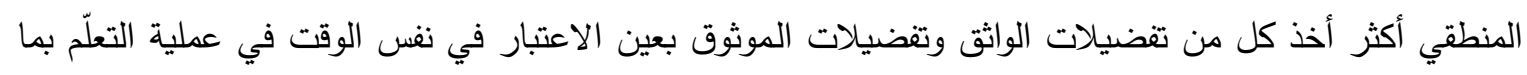

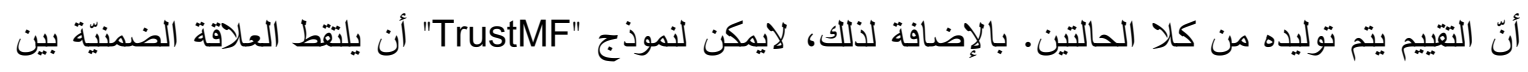

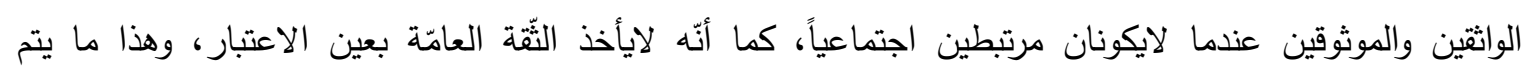
معالجته في نموذجنا المقترح.

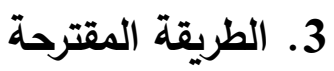

1.3

في البداية، نقدّم عدداً من الرموز المستخدمة لنمذجة مشكلة التوصية في هذا العمل. لتكن

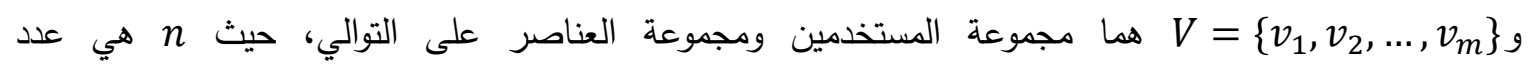
المستخدمين و m هي عدد العناصر • ولتكن المعطاة من المستخدمين على العناصر ، و $R_{i, j}$ تمثّل التقييم المعطى من المستخدم $u_{i}$ إلى العنصر

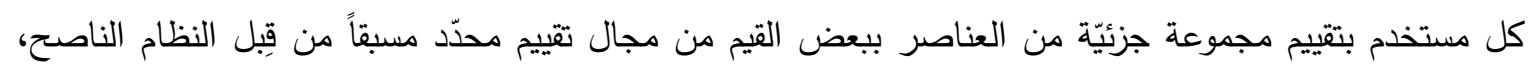
ولتكن هي مصفوفة علاقات الثّّة للمستخدم، حيث $T \in \mathbb{R}^{n \times n}$

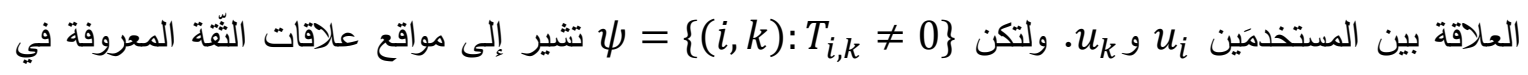
مصفوفة الثّقة T. بما أنّا نستخدم في هذا العمل تقنيّة تحليل المصفوفات في بناء النموذج المقترح، ليكن و بالنسبة للمستخدم u $u_{i}$ بالتالي يمكننا صياغة مشكلة التوصية في هذا العمل كمايلي: بإعطاء مجموعة من تقييمات المستخدمين على تلى

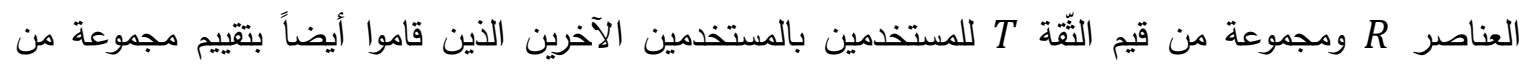
العناصر ، وباستخدام تقنيّة تحليل المصفوفات نريد تقصيّ كيفيّة تعلّم تفضيلات الحالات المختلفة للمستخدمين ومزايا

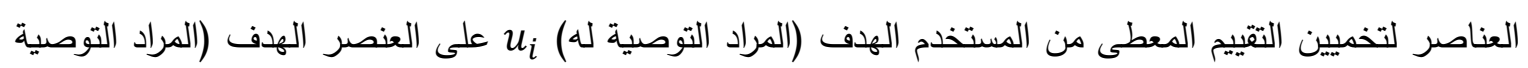

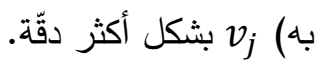


2.3 نموذج تحليل المصفوفات إلى عوامل يفترض نموذج تحليل المصفوفات إلى عوامل أنّ القليل من العوامل الكامنة تؤثّر على سلوكيات التقييم للمستخدم وأنّ شعاع تفضيلات المستخدم يتم تحديده بكيفيّة تطبيق كل عامل على ذلك المستخدم [13]. هذه الفرضيّة تجعل من المككن اكتثاف التقييمات المفقودة في مصفوفة التقييم من التقييمات المعروفة بثكل فعّال. تقوم هذه التقتيّة

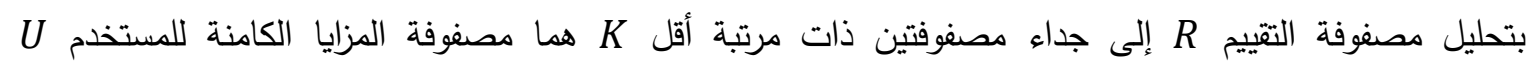

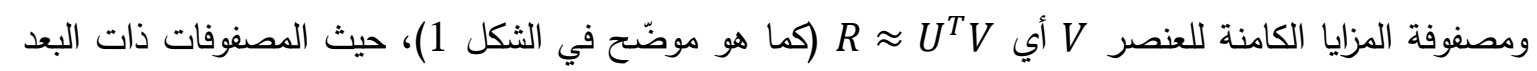
المنخفض U U V غير معروفة وتحتاج لأن يتم تخمينها. بالتالي الهدف من تقنيّة تحليل المصفوفات هو تعلّم مصفوفات المزايا الكامنة U و V وتوظيفها لاحقاً لتقديم تتبؤات بالتقييمات المفقودة [14] وذللك من خلال حل مشكلة التحسين التاليّة:

$$
\text { (1) } \min _{\mathrm{U}, \mathrm{V}} \sum_{i=1}^{n} \sum_{j=1}^{m} W_{i, j}\left(R_{i, j}-U_{i}^{T} V_{j}\right)^{2}+\lambda\left(\|U\|_{F}^{2}+\|V\|_{F}^{2}\right)
$$

حيث يمثّل

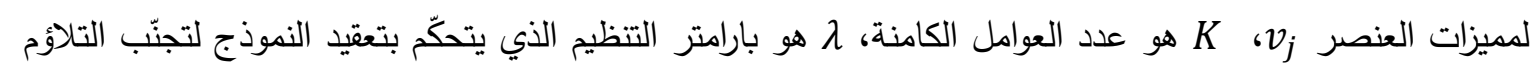

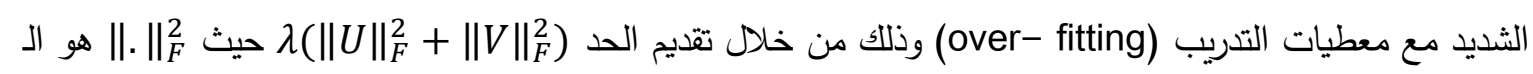

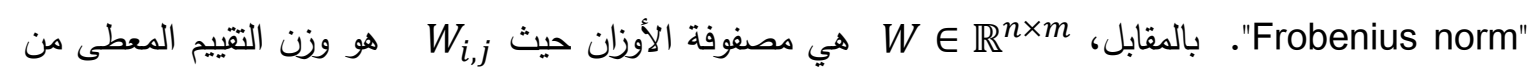
المستخدم $u_{i}$ استخدام مصفوفة الأوزان W لمعالجة الآراء الضمنيّة أيضاً وترميز المعلومات الجانبيّة مثل التشابه بين المستخدمين

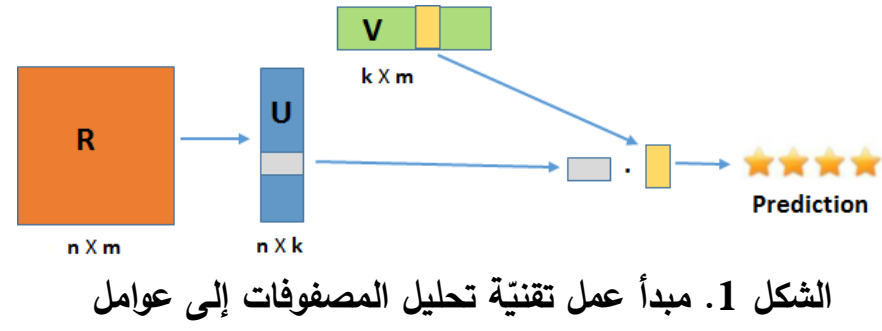

إنّ مشكلة التحسين السابقة تسعى لتقليل المجموع التربيعيّ للأخطاء بين القيم الحقيقيّة والقيم المتنبًأ بها بحدود

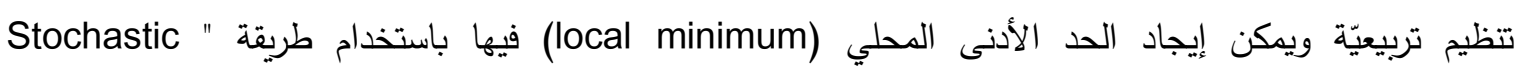
Gradient Descent المستخدم $u_{i}$ . $\widehat{R}_{i, j}=U_{i}^{T} V_{j} V_{j}$ 3.3

1.3.3 نمذجة المعلومات المحليّة في الشبكات الاجتماعية

في طريقتا المقترحة، تمثّل المعلومات المحليّة تفضيلات الحالتين المختلفتين للمستخدم (كواثق وكموثوق به) والتي يتم تعلّمها عن طريق نمذجة التفاعلات الصريحة والتفاعلات الضمنيّة بين المستخدمين. على وجه التحديد، يتم تخمين التفضيلات الخاصّة بالواثث والتفضيلات الخاصّة بالموثوق بحيث تلائم التقييمات الصريحة وعلاقات الثّقة

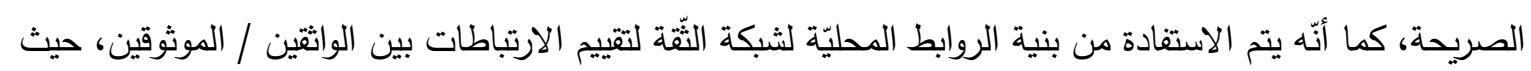

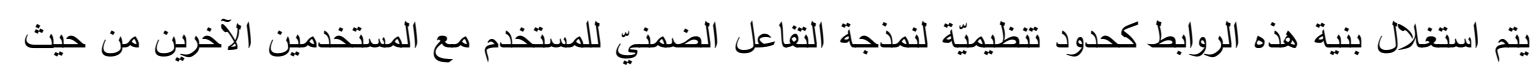
التفضيلات الخاصّة بالواثثين والتفضيلات الخاصّة بالموثوقين. 
a نصف في هذا المقطع كيفيّة توليد التقييمات وعلاقات الثّقة من وجهة نظر الحالات المختلفة للمستخدم كما هو

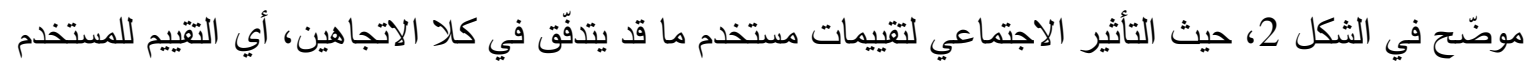

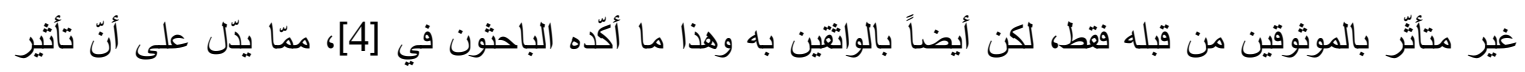

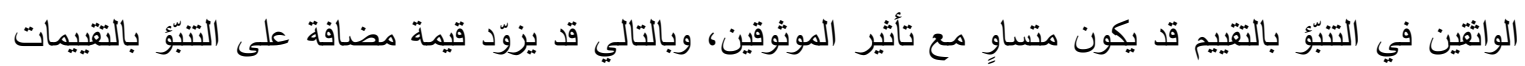
بشكل أكثر دقّة. نمنجة التقييم نظراً لفرضيّة أنّ الحالات المختلفة للمستخدم تئّرّر على توليد التقييم بطريقة مختلفة [4]، فإنّ كل تقييم معروف يجب تحديده بواسطة التفضيلات الخاصّة بالواثق وكذلك التفضيلات الخاصّة بالموثوق. اعتماداً على ذلك، يتم التبنوّ بالتقييم من المستخدم $u_{i}$

$$
\text { (2) } \hat{R}_{i, j}=\alpha B_{i}^{T} V j+(1-\alpha) E_{i}^{T} V_{j}
$$

حيث $\alpha$ هو باراميتر للتحكّم بمساهمة التقييم من الحالات المختلفة للمستخدم. بالتالي بإعطاء معطيات التقييم

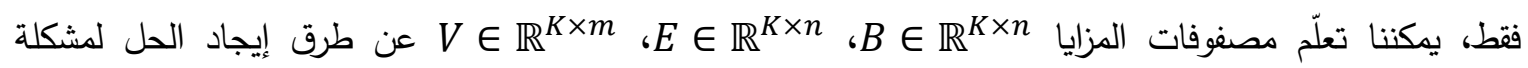

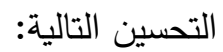

$$
+\lambda\left(\|B\|_{F}^{2}+\|E\|_{F}^{2}+\|V\|_{F}^{2}\right) \quad(3) \min _{B, E, V} \sum_{(i, j) \in \Omega}\left(R_{i, j}-g\left(\alpha B_{i}^{T} V j+(1-\alpha) E_{i}^{T} V_{j}\right)\right)^{2}
$$

حيث (x) هو التابع اللوجستي المقترح من قِبل الباحثين في [16] لتقييد الجداء الداخلي لأشعّة المزايا الكامنة في المجال [0,1 10 ويُعطى بالعلاقة

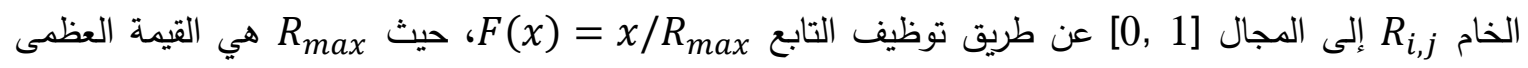

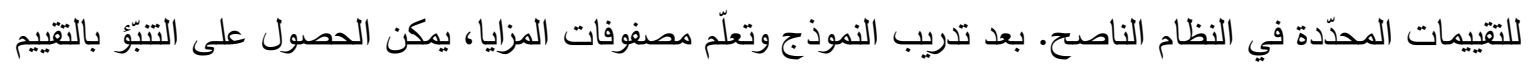

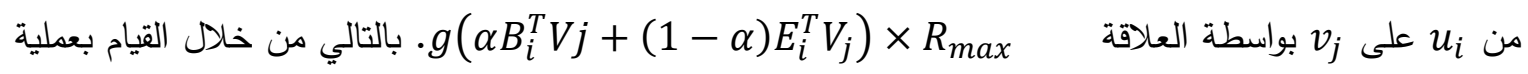
تحليل المصفوفات السابقة، يمكن للشخص تعلّم تفضيلات المستخدم من حيث أشعّة المزايا الكامنة الخاصّة بالمستخدم بحالتيه كواثق وكموثوق من معطيات التقييم المعروفة.

نمذجة الثقة

بالإضافة لمعطيات التقييم، تكون هناك شبكة ثقة ضخمة مولّدة من قِبل المستخدمين متاحة أيضاً في مواقع

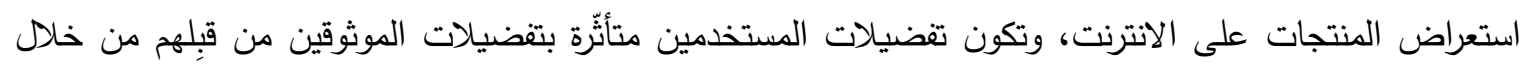

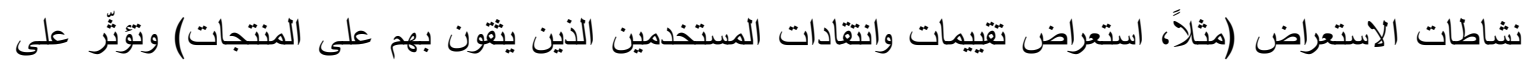

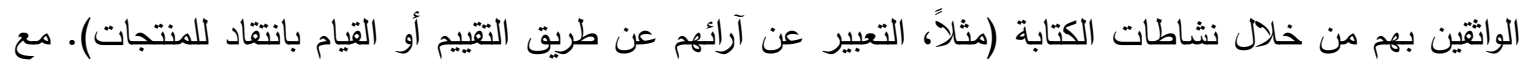

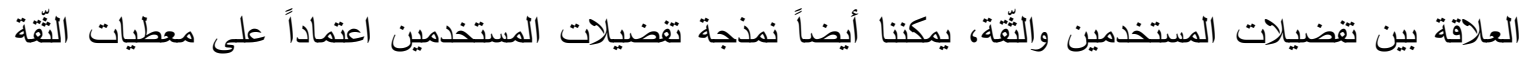

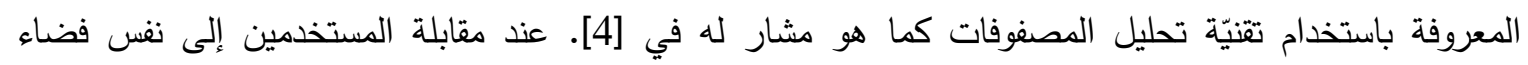

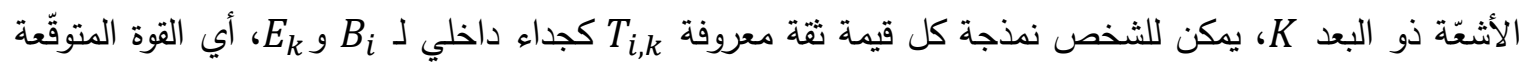
لعلاقة الثّقة بين u و $u_{i}$ و تُعطى كمايلي:

$$
\text { (4) } \widehat{T}_{i, k}=B_{i}^{T} E_{k}
$$

بإعطاء معطيات الثّّة فقط، يمكننا تعلم مصفوفات المزايا لمشكلة التحسين التالية: باءع معطيات 


$$
(5) \min _{B, E} \sum_{(i, k) \in \psi}\left(T_{i, k}-g\left(B_{i}^{T} E_{k}\right)\right)^{2}+\lambda\left(\|B\|_{F}^{2}+\|E\|_{F}^{2}\right)
$$

بالتالي من خلال القيام بعملية تحليل المصفوفات السابقة، يمكن للشخص تعلّم تفضيلات المستخدمين من حيث أشعّة

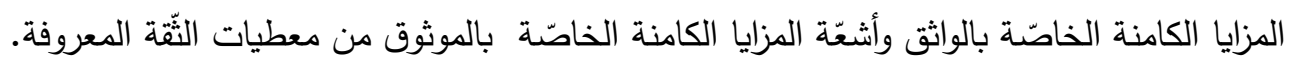

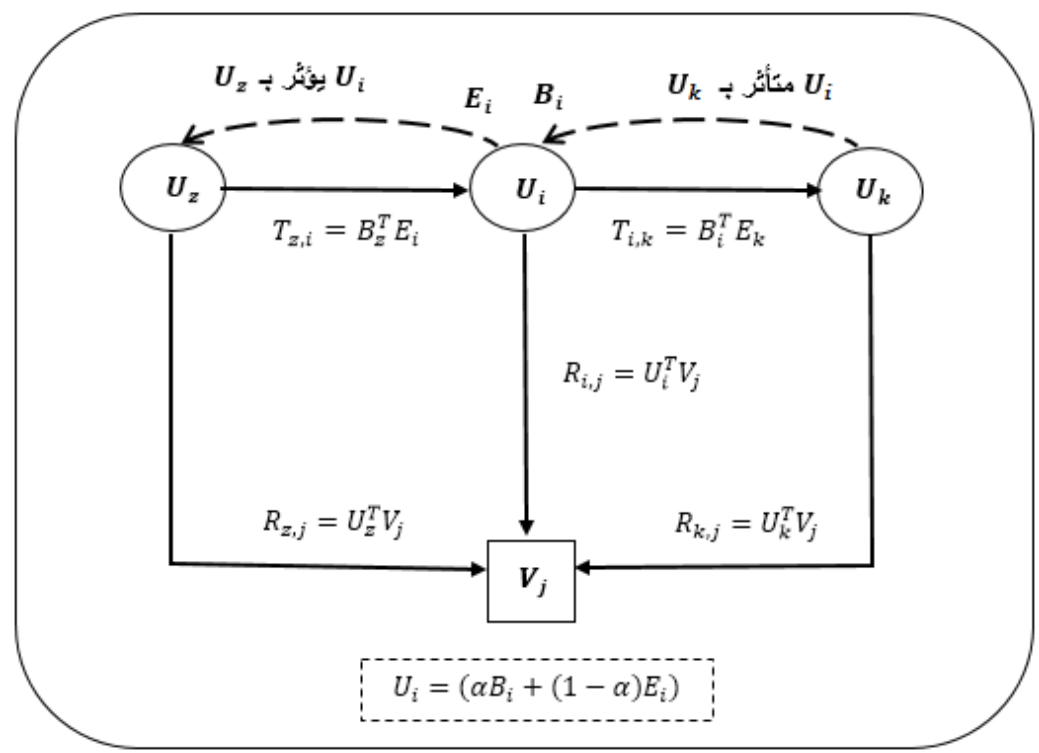

الثكل 2. تأثير الواثقين والموثوقين على التنبّوّ بالتقييم بالنسبة للمستخدم الهدف على العنصر الهدف b نمذجة التفاعلات الضمنيّة بين المستخدمين التين نصف في هذا المقطع كيفيّة نمذجة التفاعلات الضمنيّة بين اثثين من الواثثين/ الموثوقين عن طريق دمج بنية

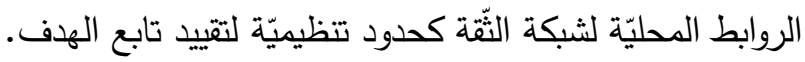
التأثير الضمني للواثقين يكون اثنان من المستخدمين الواثقين متثابهين إذا تشاركا العديد من الروابط الخارجة (out-links) في شبكة الثّّة، أي أنّهما يثقان بشكل مشترك بالعديد من المستخدمين الموثوقين. بالتالي عن طريق أخذ كل روابط الثّّة للمستخدم بالمستخدمين الآخرين وبدلاً من الاعتماد على رابط وحيد فقط، يمكننا الحصول على ارتباط أكثر دقّة

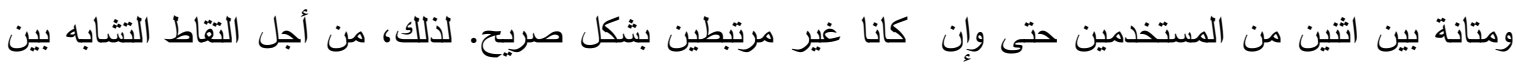

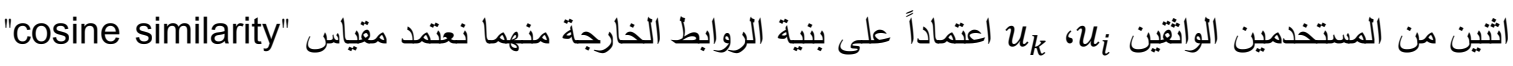

$$
\text { (6) } S_{i, k}^{B}=\frac{\sum_{f=1}^{n} T_{i, f} \cdot T_{k, f}}{\sqrt{\sum_{f=1}^{n} T_{i, f}^{2}} \sqrt{\sum_{f=1}^{n} T_{k, f}^{2}}}
$$

حيث في تجاربنا، استخدمنا قيمة ثنائية للثّّة

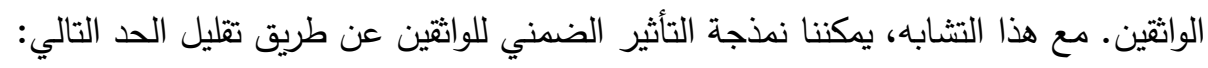
(7) $\sum_{i=1}^{n} \sum_{k=1}^{n} S_{i, k}^{B}\left\|B_{i}-B_{k}\right\|_{F}^{2}$

إنّ القيمة الكبيرة لـ $S_{i, k}^{B}$ تشير إلى أن الواثق u $u_{i}$

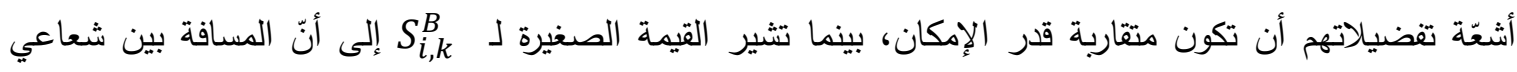


التضضيل يجب أن تكون كبيرة. بالتالي من خلال تقديم هذا التشابه المعتمد على البنية، فإنّ أشعّة التضضيلات الخاصّة بالواثقين يتم تقييدها في عملية التعلّم. التأثير الضمني للموثوقين يكون اثنان من المستخدمين الموثوقين متشابهين إذا تثاركا العديد من الروابط الداخلة (in-links) في شبكة الثّّة، أي هما موثوقان بشكل مشترك من قبل العديد من المستخدمين الواثقين. بالتالي يمكن التقاط التشابه بين اثثين من التن

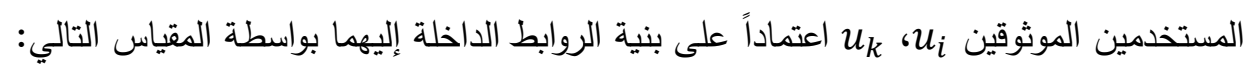

$$
\text { (8) } S_{i, k}^{E}=\frac{\sum_{f=1}^{n} T_{f, i} \cdot T_{f, k}}{\sqrt{\sum_{f=1}^{n} T_{f, i}^{2}} \sqrt{\sum_{f=1}^{n} T_{f, k}^{2}}}
$$

حيث تشير E هنا إلى التشابه بين اثثين من الموثوقين. بشكل مشابه لنمذجة التأثر الضمني للواثتين، نقوم بنمذجة التأثير الضمني للموثوقين عن طريق تقليل الحد التالي:

$$
\text { (9) } \sum_{i=1}^{n} \sum_{k=1}^{n} S_{i, k}^{E}\left\|E_{i}-E_{k}\right\|_{F}^{2}
$$

\subsection{3 نمذجة المعلومات العامّة في الشبكات الاجتماعيّة}

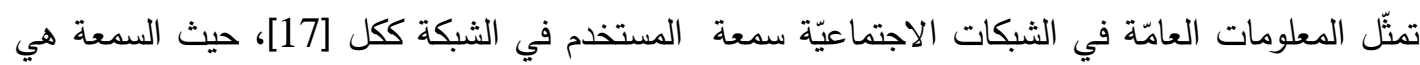
نوع من الحالة التي تعطي قوى وإمكانيّات إضافيّة في النظم الناصحة [12]. هنالك العديد من الخوارزميّات لحساب فئي قيمة السمعة للعقد في الثبكات الاجتماعيّة وفقاً لارتباطاتهم [18]، [19]. نعتمد في هذا فئهي العمل إحدى الخوارزميات الأكثر شعبيّة وهي "PageRank" [18] لحساب قيم السمعة للمستخدمين، حيث نقوم أولاً بتطبيق خوارزمية

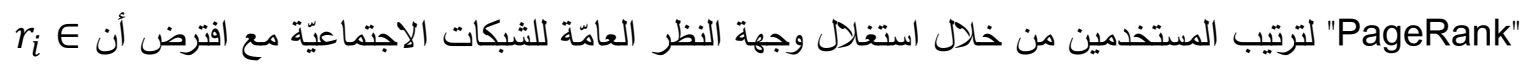

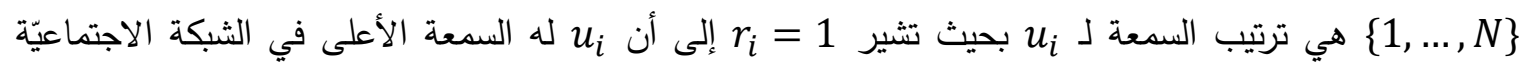
الكاملة، من ثمّ نعرّف قيمة السمعة

$$
(10) w_{i}=f\left(r_{i}\right)=\frac{1}{1+\log \left(r_{i}\right)}
$$

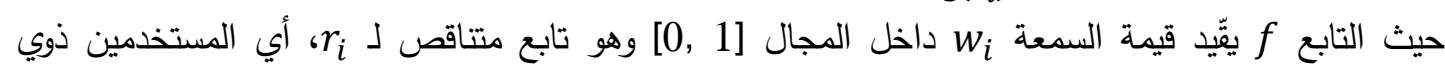
الترتيب الأعلى لهم قيم سمعة عالية. بالتالي لنمذجة المعلومات العامّة في الشبكات الاجتماعيّة، يمكننا استخدام قيم سمعة المستخدم لتوزين أهميّة توصياتهم بتعديل المعادلة (3) السابقة لتصبح كمايلي: $+\lambda\left(\|B\|_{F}^{2}+\|E\|_{F}^{2}+\|V\|_{F}^{2}\right)(11) \min _{B, E, V} \sum_{(i, j) \in \Omega} w_{i}\left(R_{i, j}-g\left(\alpha B_{i}^{T} V j+(1-\alpha) E_{i}^{T} V_{j}\right)\right)^{2}$

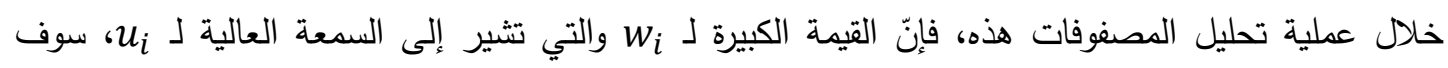

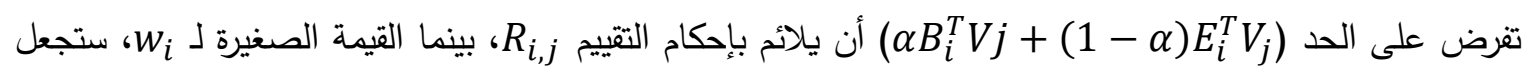
الحد 3.3.3 إطار العمل الموحّد للنموذج المقترح حتى الآن، عرضنا كيفيّة نمذجة التقاعلات الصريحة (من حيث التقييم والثّقة) والتفاعلات الضمنيّة بين الواثتين

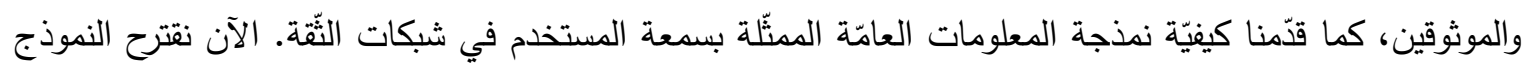
المدموج التالي الذي يأخذ كل المعلومات السابقة بعين الاعتبار ويقوم بإيجاد الحل لتابع الهدف التالي:

$$
\begin{gathered}
\mathcal{L}=\frac{1}{2} \sum_{(i, j) \in \Omega} w_{i}\left(R_{i, j}-g\left(\alpha B_{i}^{T} V j+(1-\alpha) E_{i}^{T} V_{j}\right)\right)^{2} \\
+\frac{1}{2} \sum_{(i, k) \in \psi}\left(T_{i, k}-g\left(B_{i}^{T} E_{k}\right)\right)^{2}
\end{gathered}
$$




$$
\begin{array}{r}
+\frac{\lambda_{B}}{2} \sum_{i=1}^{n} \sum_{k=1}^{n} S_{i, k}^{B}\left\|B_{i}-B_{k}\right\|_{F}^{2} \\
+\frac{\lambda_{E}}{2} \sum_{i=1}^{n} \sum_{k=1}^{n} S_{i, k}^{E}\left\|E_{i}-E_{k}\right\|_{F}^{2} \\
+\frac{\lambda}{2}\left(\|B\|_{F}^{2}+\|E\|_{F}^{2}+\|V\|_{F}^{2}\right)
\end{array}
$$

حيث

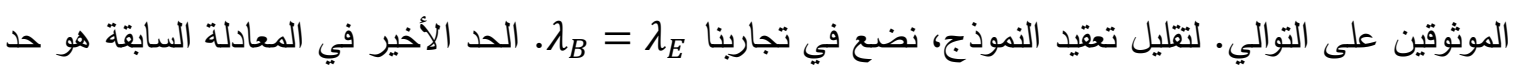
التظيم المستخدم لتجنّب التلاؤم الثديد مع معطيات التدريب و $\lambda$ هو بارامتر التظيم. 4.3

للحصول على الدد الأدنى الأصغري لتابع الهدف السابق في المعادلة (12) من أجل تعلّم مصفوفات المزايا B، E، V واستخدامها للتنبّؤ بالتقييمات غير المعروفة، نستخدم طريقة "SGD"، والتي تعمل عادةً بشكل جيد بالنسبة للنظم الناصحة. يتم إعطاء الخوارزميّة الممثّة لتعلّم النموذج وتعديله في الخوارزمية 1. هناك عدّة بارامترات تمّ أخذها كدخل،

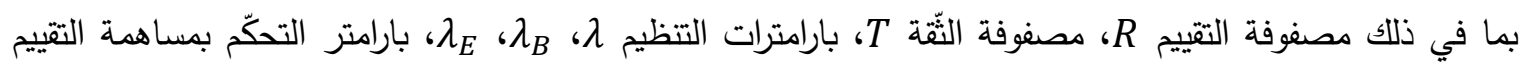

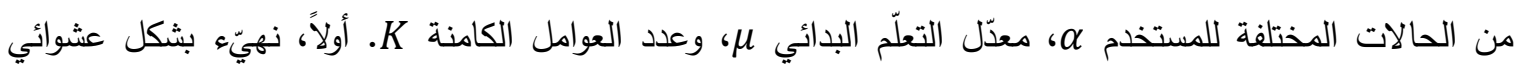

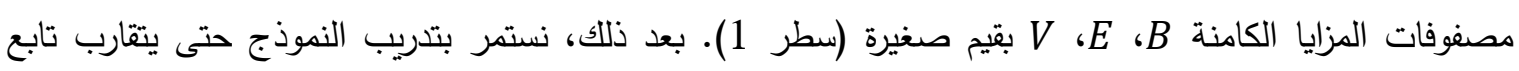
الهدف L (سطر 2). على وجه التحديد، نحسب مشتقات المتحولات B، E، V V وبعدها نعدّل هذه المتحولات باستخدام

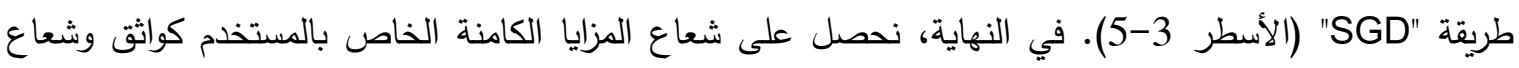
المزايا الكامنة الخاص بالمستخدم كموثوق وشعاع المزايا الكامنة الخاص بالعنصر ونستخدمها في حساب التنبؤ من المستخدم الهدف على العنصر الهدف.

\section{الخوارزمية 1 خوارزمية التعلّم من أجل النموذج المقترح}

\section{Algorithm}

Input: $R, T, K, \lambda, \lambda_{B}, \lambda_{E}, \alpha$, $\mu$ (learning rate)

Output: $B, E, V$

1. Initialize $B, E, V$ with small random numbers

2. While $\mathcal{L}$ has not converged:

3. $B_{i} \leftarrow B_{i}-\mu \cdot \frac{\partial \mathcal{L}}{\partial B_{i}}, i=1, \ldots, n$

4. $E_{i} \leftarrow E_{i}-\mu \cdot \frac{\partial \mathcal{L}}{\partial E_{i}}, i=1, \ldots, n$

5. $V_{j} \leftarrow V_{j}-\mu \cdot \frac{\partial \mathcal{L}}{\partial V_{j}}, j=1, \ldots, m$

6. End while

The predict rating:

$\hat{R}_{i, j}=g\left(\alpha B_{i}^{T} V j+(1-\alpha) E_{i}^{T} V_{j}\right) \cdot R_{\max }$ 
نختار مجموعة معطيات "Epinions" كمصدر معطيات لتجاربنا، والتي تحوي معلومات عن تقييمات المستخدمين على العناصر ، ومعلومات عن شبكة الثّّة بين المستخدمين وهي المعلومات اللازمة للتحقّق من نموذجنا.

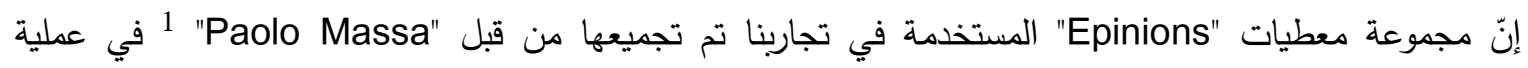

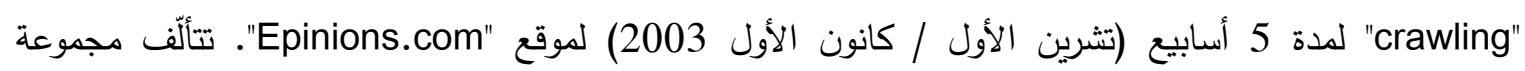
المعطيات هذه من 49,290 مستخدم والذين قاموا بتقييم 139,738 عنصر مختلف مرّة واحدة على الأقل، من بينهم

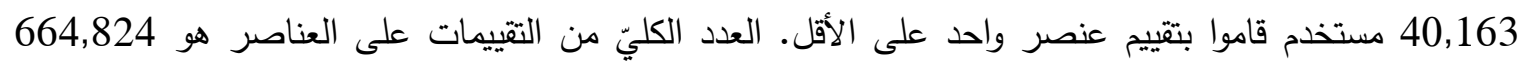
تقييم، والعدد الكليّ من تصريحات الثّّة على المستخدمين هو 487,183 تتيبم ثقة والتي تم إصدارها من قبل

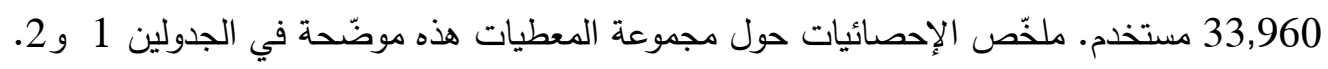

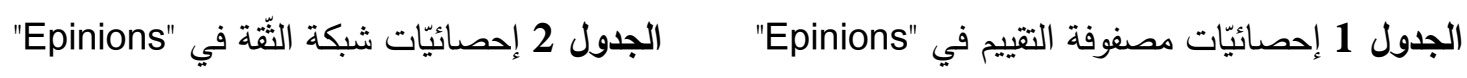

\begin{tabular}{|c|c|c|c|}
\hline 487,183 & عدد علاقات الثّقة & 49,290 & عدد المستخدمين \\
\hline 33,960 & عدد المستخدمين الواثثين & 139,738 & عدد العناصر \\
\hline 49,288 & عدد المستخدمين الموثوقين & 664,824 & عدد التقييمات \\
\hline \multirow{2}{*}{$\begin{array}{c}0.029 \\
\%\end{array}$} & \multirow[t]{2}{*}{ نسبة كثافة علاقات الثّقة } & 40,163 & عدد المستخدمين الذين لهم تقييمات \\
\hline & & $\% 0.051$ & نسبة كثافة المعطيات \\
\hline 1,760 & العدد الأعظمي من الواثقين لكل مستخدم & \multirow[t]{2}{*}{1,023} & \multirow{2}{*}{ العدد الأعظمي من التقييمات من قِبل } \\
\hline \multirow[t]{2}{*}{2,589} & \multirow{2}{*}{ مستخدم العدد الأعظمي من الموثوقين لكل } & & \\
\hline & & \multirow[t]{2}{*}{2,026} & \multirow{2}{*}{ العدد الأعظمي من التقييمات على } \\
\hline 14.35 & العدد الوسطي من الواثتين لكل مستخدم & & \\
\hline \multirow[t]{4}{*}{9.88} & العدد الوسطي من الموثوقين لكل مستخدم & \multirow[t]{2}{*}{16.55} & \multirow{2}{*}{ العدد الوسطي من التقييمات من قِبل } \\
\hline & & & \\
\hline & & 4.76 & العدد الوسطي من التقييمات على \\
\hline & & {$[5 ، 1]$} & 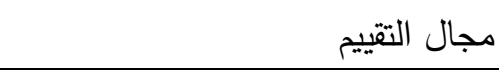 \\
\hline
\end{tabular}

لاحظنا أيضاً من خلال مجموعة من التجارب التي تّّ إجراؤها أنّ توزيعات المستخدمين الموثوقين والواثقين لمجموعة معطيات "Epinions" تلائم توزيع "power-law"، كما هو الحال في العديد من الشبكات الاجتماعيّة، وهذا موضّح في الثكلين 3 و4 على التوالي، حيث القليل فقط من المستخدمين الموثوقين لديهم العديد من المستخدمين الواثثين بينما معظم المستخدمين الموثوقين لديهم عدد قليل فقط من المستخدمين الواثثين، الأمر نفسه ينطبق بالنسبة للمستخدمين الواثقين. هذا يدل على وجود بعثرة كبيرة في معطيات الثقة المزودة من المستخدمين.

1 http://www.trustlet.org/downloaded_epinions.html 


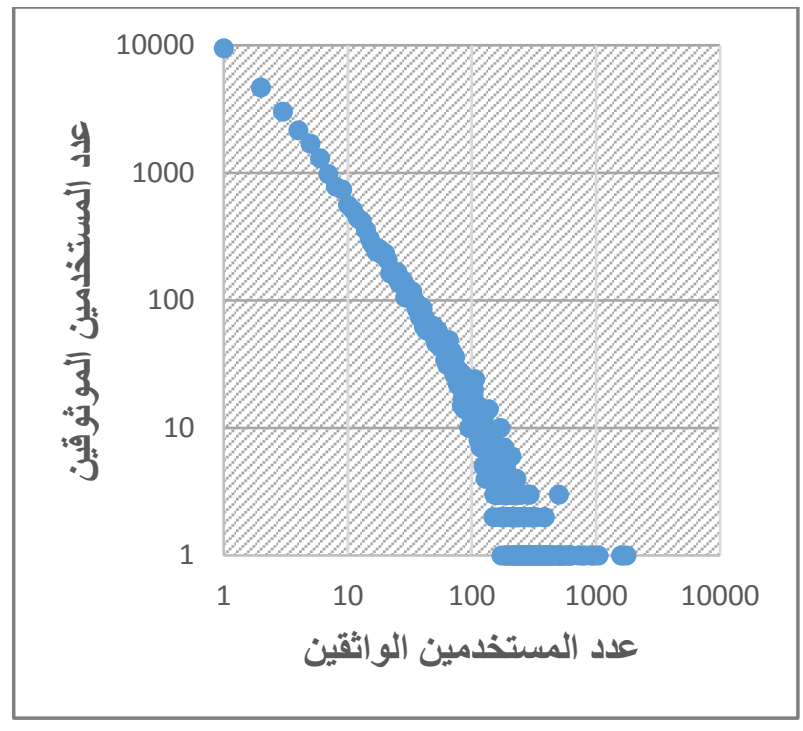

الشكل 4. توزيع المستخدمين الواثقين بالنسبة للمستخدمين الموثوقين

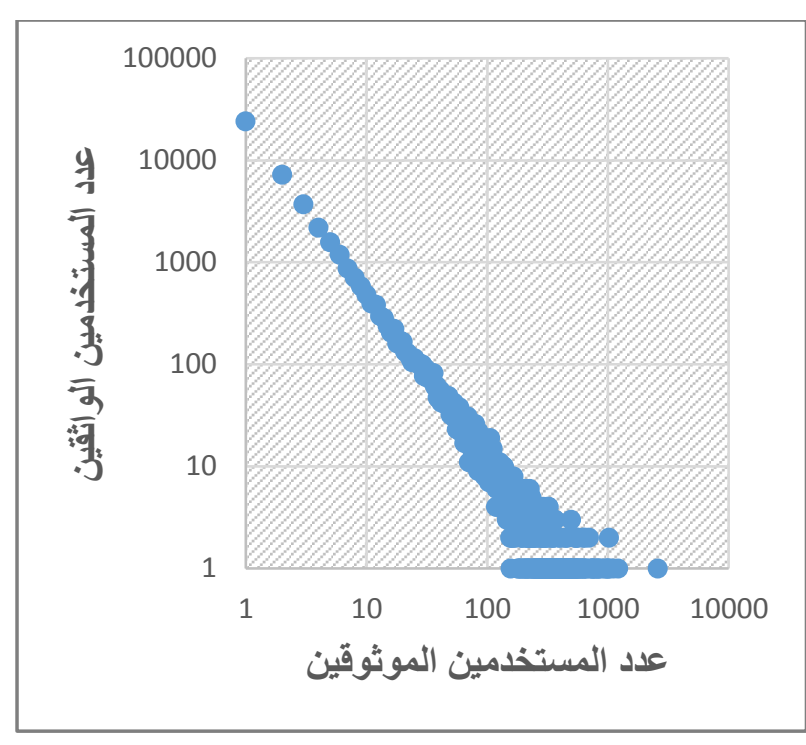

الشكل 3. توزيع المستخدمين الموثوقين بالنسبة للمستخدمين الواثقين

2.4 لقياس جودة التتبّوّ للطريقة المقترحة مقارنةً مع طرق التصفية التعاونيّة والطرق الأخرى المعتمدة على الثّقة، نستخدم مقياسين شائعين هما [2]: مقياس "Mean Absolute Error) "MAE): وهو يقيس متوسّط الفروقات المطلقة بين التقييمات الفعليّة الصحيحة $R_{i, j}$ المعطاة من قِبل المستخدم والتقييمات المتنبأ بها من قِبل النظام الناصح (13)MAE $=\frac{1}{N} \sum_{\left(u_{i}, \mathrm{v}_{j}\right) \in \text { TestSet }}\left|\hat{R}_{i, j}-R_{i, j}\right|$

حيث N هي عدد التقييمات التي نريد اختبارها، وكلّما كانت قيمة مقياس "MAE" أقل كلّما كان التنبّوّ أفضل. مقياس "Root Mean Absolute Error)"RMSE): وهو يقيس الجذر التربيعيّ لمتوسّط مربّع الفروقات بين التقييمات الصحيحة Ri,j المعطاة من قبل المستخدم والتقييمات المتنبأ بها من قبل النظام الناصح بالعلاقة التالية:

$$
\text { (14)RMSE }=\sqrt{\frac{1}{N} \sum_{\left(u_{i}, \mathrm{v}_{j}\right) \in T e s S e t}\left(\hat{R}_{i, j}-R_{i, j}\right)^{2}}
$$
كذلك كلّما كانت قيمة مقياس "RMSE" أقل كلّما كان التنبّوّ أفضل.

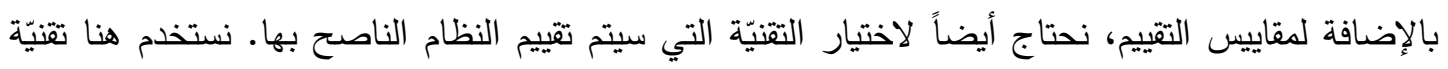
تصربأfold cross-validation" تدريب لتدريب النموذج ومجموعة اختبار لتقييمه. على وجه التحديد، يتم تقسيم مجموعة المعطيات بشكل عشوائي إلى ولى لتئي 5 أجزاء، من بين الأجزاء الخمسة، يتم إبقاء جزء واحد كمعطيات اختبار، والأجزاء الأربعة المتبقية يتم استخدامها كمعطيات تدريب. يتم تكرار هذه العملية 5 مرات، مع استخدام كل واحد من الأجزاء الخمسة كمعطيات اختبار مرّة واحدة فقط، وفي النهاية يتم أخذ متوسّط النتائج لإعطاء النتيجة النهائية. في تجاربنا، نستخدم في كل جزه 
المعطيات كمجموعة تدريب والـ 20\% المتبقية كمجموعة اختبار ، والذي يعني أنتّا نختار عشوائياً 80\% من التقييمات

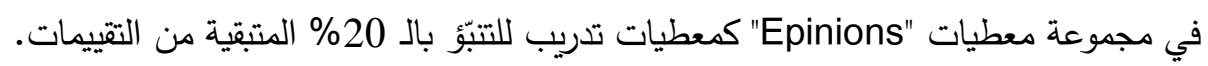

3.4 الإعدادات التجريبيّة

لإظهار تحسينات الأداء لطريقتا المقترحة، نقوم بمقارنتها مع طريقة تحليل المصفوفات التقليديّة ومع عدد من الطرق المعتمدة على الثّّة الموجودة في الدراسات السابقة والأقرب إلى طريقتيا:

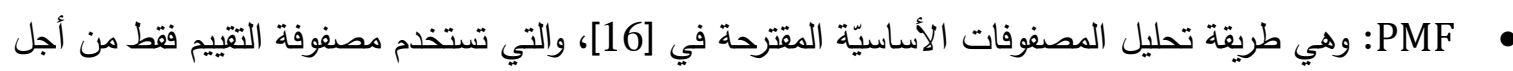
التوصيات دون أخذ العلاقات الاجتماعيّة بين المستخدمين بعين الاعتبار .

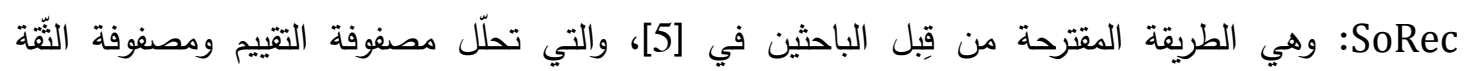
الاجتماعيّة للمستخدمين عن طريق مشاركة نفس فضاء المزايا الكامن للمستخدم.

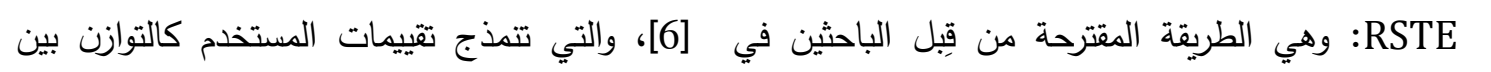
تفضيلاته الخاصّة وتفضيلات المستخدمين الموثوقين من قبله. SocialMF نمذجة علاقات الثّّة بين المستخدمين. هoReg: وهي الطريقة المقترحة من قِبل الباحثين في [8]، والتي فيها تتم نمذجة علاقات الثّّة بافتراض أنّ التّات

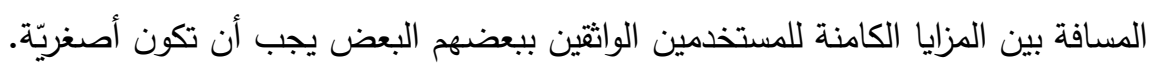
TrustMF

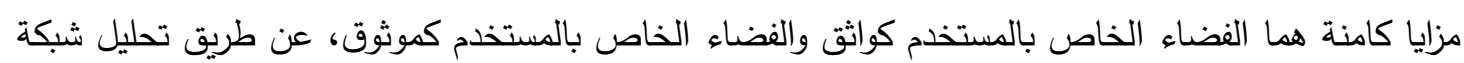
الثّّة وفقاً لخصائص الاتجاه للثقة. • وهي الطريقة المقترحة الخاصّة بنا والتي يتم فيها استغلال السياق الاجتماعي العام والمحليّ لعلاقات الثقّة بين المستخدمين، حيث تمثّل المعلومات المحليّة تفضيلات الحالتين المختلفين للمستخدمين (كواثق وكموثوق به) والتي يتم تعلّمها عن طريق نمذجة التفاعلات الصريحة والضمنيّة بين المستخدمين، بينما تمثّل

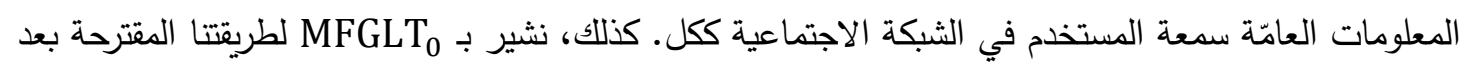

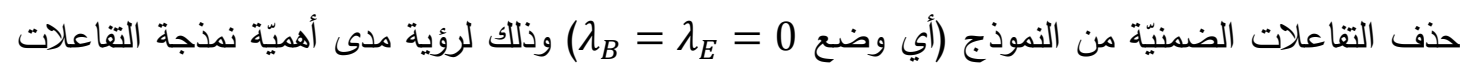
الضمنيّة في تحسين دقّة التوصيات بشكل إضافي. بالنسبة لكل الطرق التي تتم المقارنة معها، حتى يتم التحقّق منها، نضع بارامترات أمثليّة خاصّة إمّا وفقاً للمراجع

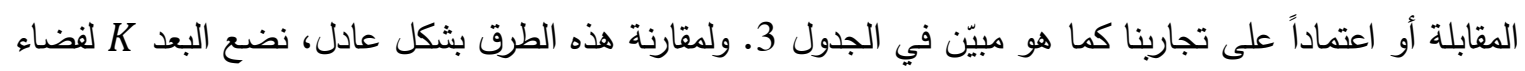

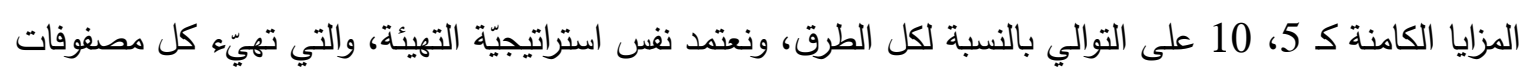

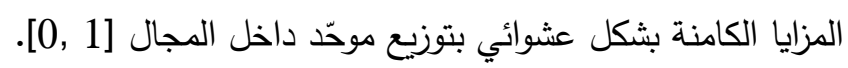


الجدول 3 إعدادات البارامترات بالنسبة لطرق المقارنة

\begin{tabular}{|c|c|}
\hline البارامترات الأمثليّة & الطرة \\
\hline$\lambda_{U}=\lambda_{V}=0.001$ & PMF \\
\hline,$\lambda_{C}=1 \lambda_{U}=\lambda_{V}=\lambda_{Z}=0.001$ & SoRec \\
\hline,$\alpha=0.4 \lambda_{U}=\lambda_{V}=0.001$ & RSTE \\
\hline,$\lambda_{T}=1 \lambda_{U}=\lambda_{V}=0.001$ & SocialMF \\
\hline,$\beta=0.001 \lambda_{1}=\lambda_{2}=0.001$ & SoReg \\
\hline,$\lambda_{T}=1 \lambda=0.001$ & TrustMF \\
\hline$, \lambda_{B}=\lambda_{E}=0, \lambda=0.001 \alpha=0.6$ & MFGLT $_{0}$ \\
\hline$, \lambda_{B}=\lambda_{E}=0.1, \lambda=0.001 \alpha=0.6$ & MFGLT \\
\hline
\end{tabular}

سنركّز في اختبارنا هذا على منظورين للمستخدمين لقياس أداء الطرق المختلفة التي تتم مقارنتها وهما: منظور "كل المستخدمين" (All Users) : وهم المستخدمين الذين لهم تقييم واحد على الأقل. منظور "المستخدمين ذوي الإقلاع البارد" (Cold-Start Users): وهم المستخدمين الذين لهم أقل من 5 تقييمات.

4.4

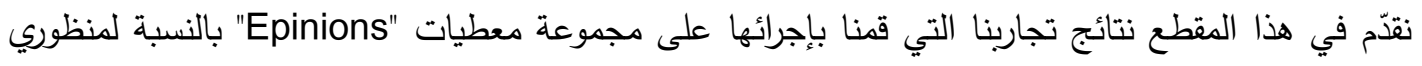
"All Users"

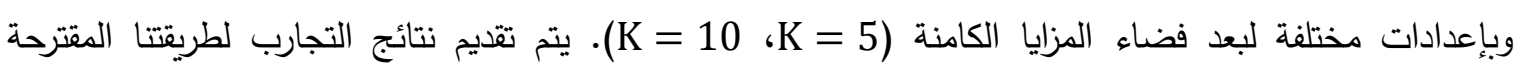
والطرق التي قمنا بالمقارنة معها في الجدول 4 فيما يتعلّق بمنظور "All Users" والجدول 5 فيما يتعلّق بمنظور "Cold-Start Users"

1.4.4 تحليل ومناقشة النتائج

في هذا المقطع، نلخّص ونناقش النتائج الأكثر أهيّة للتجارب التي تمّ القيام بها على مجموعة معطيات "Epinions" إنّ أداء طريقة "PMF" التقليديّة أدنى من أداء الطرق الأخرى قيد المقارنة المعتمدة على تحليل المصفوفات والتي تستقيد من علاقات الثّقة في منظوري "Psers" و و" All Users"

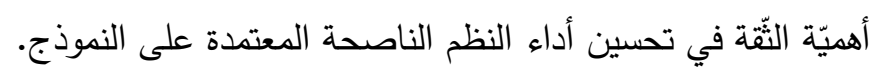

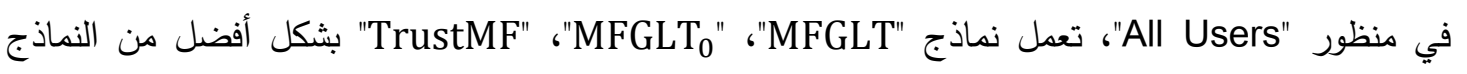
الأخرى المعتمدة على الثّّة والتي تقابل المستخدمين إلى فضاء مزايا وحيد، هذا يتطابق مع فرضيتا أنّ تمييز

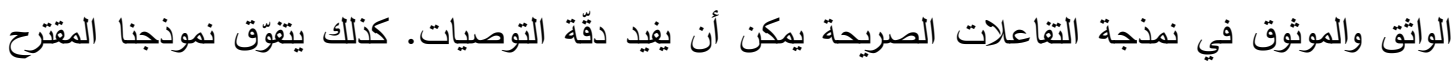

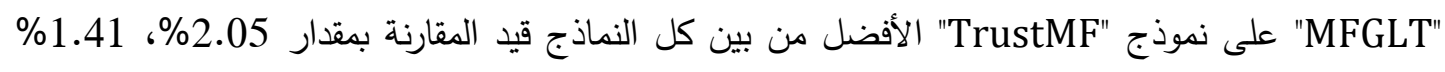

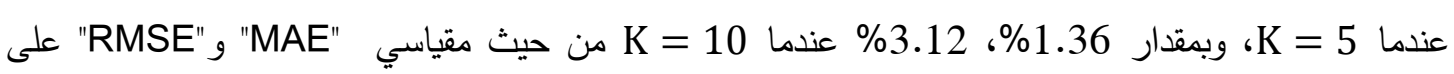
التوالي. 
في منظور "Cold-Start Users"، ليس هناك نموذج وحيد يعمل بالشكل الأفضل بالمطلق من بين النماذج

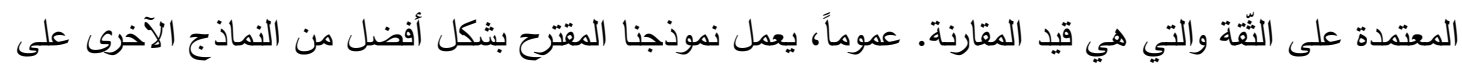

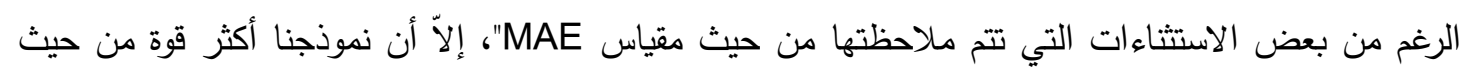

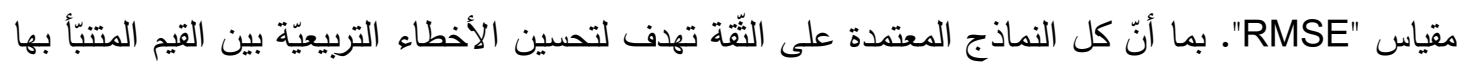

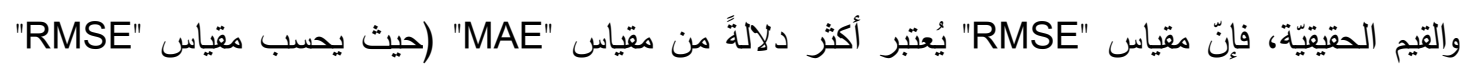

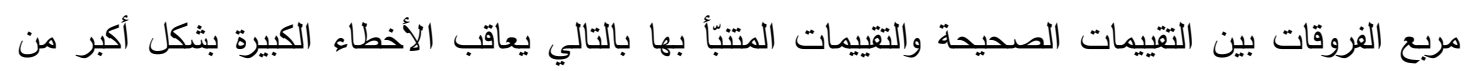

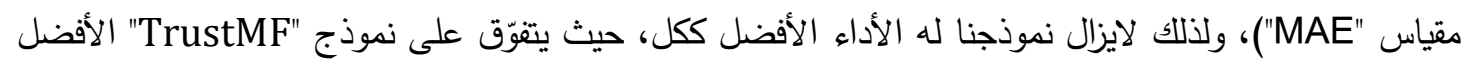

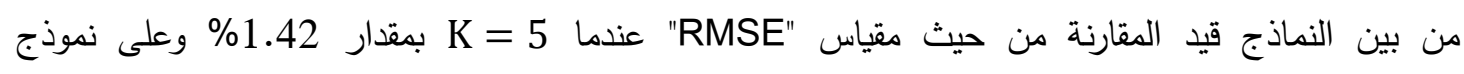

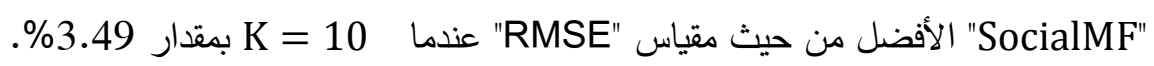

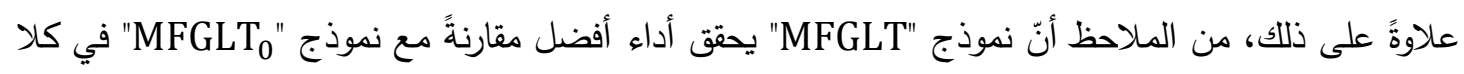

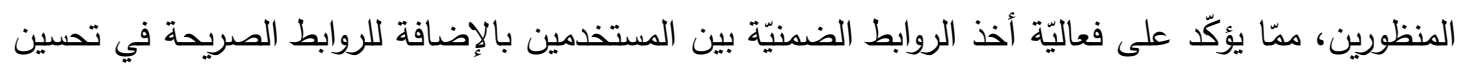

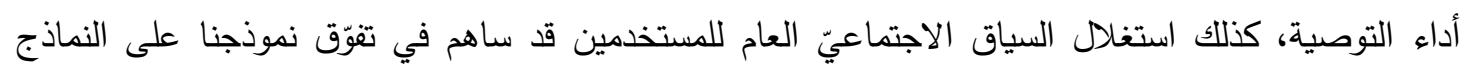
الأخرى المعتمدة على الثّّة التي تتم المقارنة معها.

على الرغم من أنّ النسبة المئويّة للتحسينات النسبيّة لنموذجنا على النماذج الأخرى قيد المقارنة صغيرة، إلاًاً أن

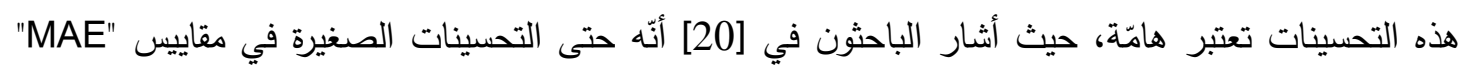
"RMSE" قد تقود إلى فروقات هامّة في التوصيات عملياً بدليل أنّه تم تقديم مبلغ مليون دولار في المنافسة

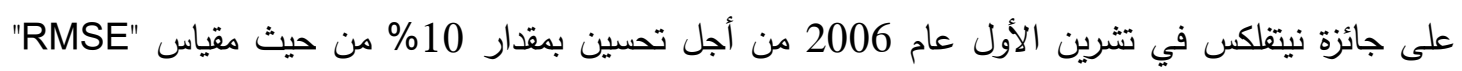
على طرق التوصية التقليديّة.

الجدول 5 الأداء التتبؤي على مجموعة معطيات

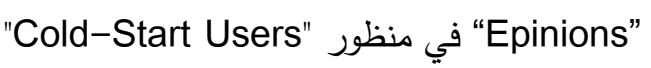

\begin{tabular}{|c|c|c|c||}
\hline \multicolumn{3}{|c|}{ “Cold-Start Users” منظو } \\
\hline \hline RMSE & MAE & K & الطريn|| \\
\hline 1.7703 & 1.4514 & 5 & PMF \\
\hline 1.4322 & 1.1531 & 10 & \\
\hline 1.1388 & 0.8929 & 5 & \multirow{2}{*}{ SoRec } \\
\hline 1.1886 & $\mathbf{0 . 8 5 1 2}$ & 10 & \\
\hline 1.1439 & 0.9143 & 5 & \multirow{2}{*}{ RSTE } \\
\hline 1.1621 & 0.8293 & 10 & \\
\hline 1.1310 & 0.8840 & 5 & \multirow{2}{*}{ SocialMF } \\
\hline 1.1512 & 0.8574 & 10 & \\
\hline 1.1888 & 0.9250 & 5 & \multirow{2}{*}{ SoReg } \\
\hline 1.2370 & 0.9833 & 10 & \\
\hline
\end{tabular}

الجدول 4 الأداء التتبؤي على مجموعة معطيات

"All Users" في منظور عبطي "Epinions"

\begin{tabular}{|c|c|c|c|}
\hline \multicolumn{4}{|c|}{ "All Users" منظور } \\
\hline RMSE & MAE & $\bar{K}$ & الطريقة \\
\hline 1.2904 & 0.9791 & 5 & \multirow[t]{2}{*}{ PMF } \\
\hline 1.1972 & 0.9093 & 10 & \\
\hline 1.1110 & 0.8807 & 5 & \multirow[t]{2}{*}{ SoRec } \\
\hline 1.1445 & 0.8848 & 10 & \\
\hline 1.1071 & 0.8635 & 5 & \multirow[t]{2}{*}{ RSTE } \\
\hline 1.1383 & 0.8572 & 10 & \\
\hline 1.0688 & 0.8245 & 5 & \multirow[t]{2}{*}{ SocialMF } \\
\hline 1.0835 & 0.8269 & 10 & \\
\hline 1.0977 & 0.8450 & 5 & \multirow[t]{2}{*}{ SoReg } \\
\hline 1.1199 & 0.8671 & 10 & \\
\hline
\end{tabular}


Journal of University of Babylon, Pure and Applied Sciences, Vol.(27), No.(1): 2019

\begin{tabular}{|c||c||c||c|}
\hline 1.1261 & 0.8910 & 5 & \multirow{2}{*}{ TrustMF } \\
\cline { 1 - 2 } 1.1763 & 0.8519 & 10 & \\
\cline { 1 - 2 } 1.1282 & 0.9007 & 5 & \multirow{2}{*}{ MFGLT $_{0}$} \\
\hline \hline 1.1776 & 0.8793 & 10 & \\
\hline \hline $\mathbf{1 . 1 1 0 1}$ & $\mathbf{0 . 8 7 6 9}$ & 5 & \multirow{2}{*}{ MFGLT } \\
\hline \hline $\mathbf{1 . 1 1 1 0}$ & 0.8776 & 10 & \\
\hline
\end{tabular}

\begin{tabular}{|c|c|c||c|}
\hline 1.0623 & 0.8178 & 5 & \multirow{2}{*}{ TrustMF } \\
\hline 1.0812 & 0.8213 & 10 & \\
\hline 1.0707 & 0.8275 & 5 & \multirow{2}{*}{ MFGLT $_{0}$} \\
\hline \hline 1.0945 & 0.8487 & 10 & \\
\hline \hline $\mathbf{1 . 0 4 7 3}$ & $\mathbf{0 . 8 0 1 0}$ & 5 & \multirow{2}{*}{ MFGLT } \\
\hline $\mathbf{1 . 0 4 7 5}$ & $\mathbf{0 . 8 1 0 1}$ & 10 & \\
\hline
\end{tabular}

\subsection{4 تأثير السياق الاجتماعي العام والمحلي على النموذج المقترح}

بيّنت النتائج التجريبيّة أنّ نموذجنا المقترح "MFGLT" يتفوّق على النماذج الأخرى المعتمدة على الثّّة كونه يستغل السياق الاجتماعيّ العام والمحليّ للمستخدمين في نفس الوقت. نتقصّى في هذا المقطع تأثير كلا السياقين على أداء النموذج المقترح عن طريق تعريف المتغيرات التالية عن النموذجئ فئاعئ

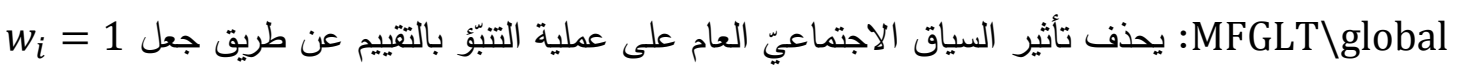

$$
\text { . } R_{i, j} \neq 0 \text { عندما }
$$

:MFGLT\local بين المستخدمين. هFGLT\global\local هذا المتغير هو نموذج تحليل المصفوفات التقليديّ "PMF".

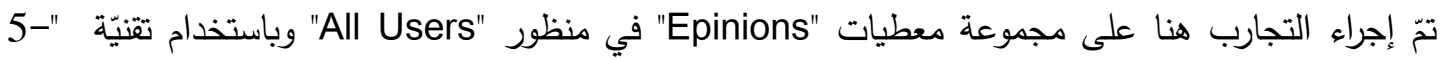

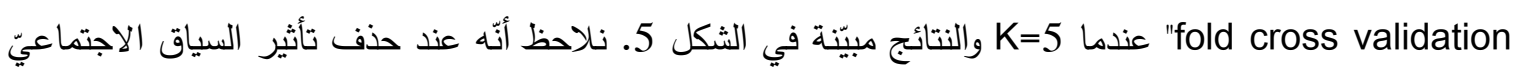

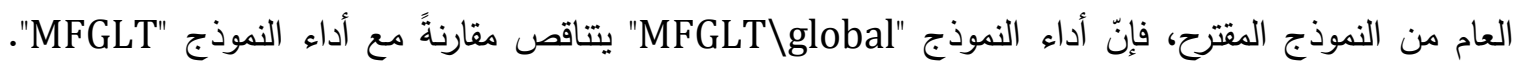
كذللك نلاحظ نفس الأمر بالنسبة لنموذج "MFGLT\local" عند حذف تأثير السياق الاجتماعيّ المحليّ. حيث مثلاً،

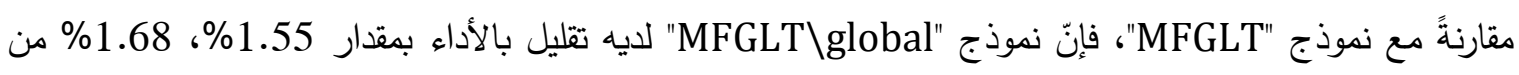

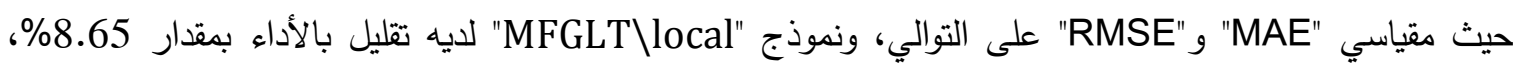
6.81\% من حيث مقياسي "MAE" و "RMSE" على التوالي. كذلك عند حذف تأثيرات السياق الاجتماعي العام والمحلي معاً في النموذج "MFGLT\global\local" نحصل على أداء أسوء من أداء نموذجي "MFGT"

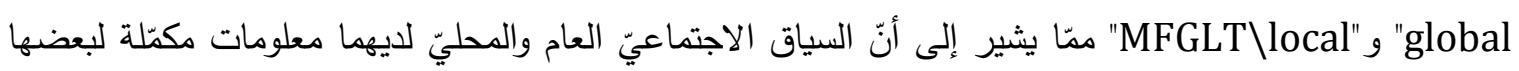
البعض وكلاهما يساهم في تحسين أداء النظام الناصح بشكل إضافيّ. 


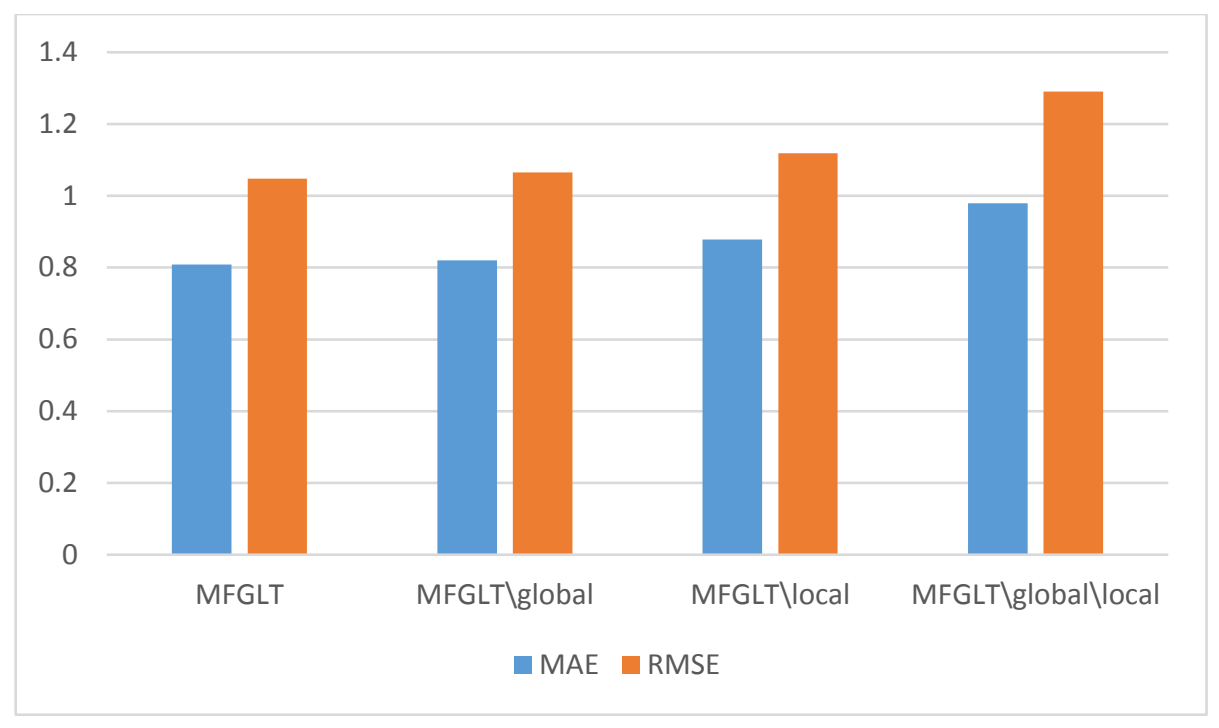

الشكل 5. أداء المتغيرات المختلفة للنموذج المقترح

3.4.4 التحقّق من أداء النموذج المقترح على مستخدمين بلرجات مختلفة من علاقات الثقة

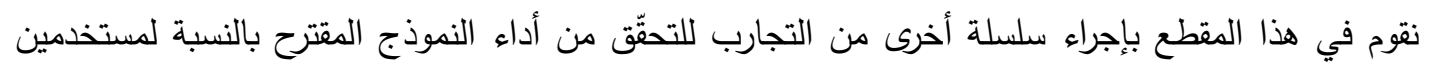
بدرجات مختلفة من علاقات الثّّة، وذلك لدقارنة طريقتا بشكل إضافيّ مع الطرق الأخرى المعتمدة على الثّّة

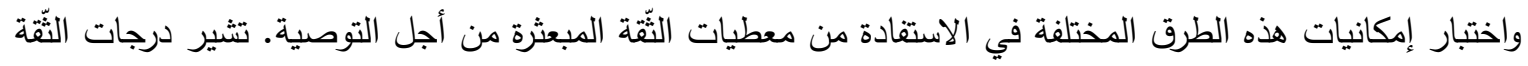

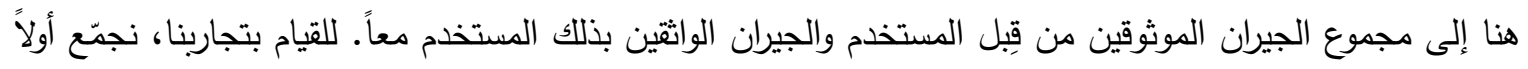

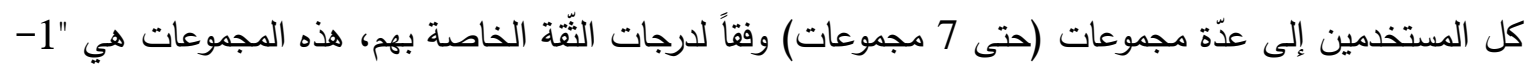

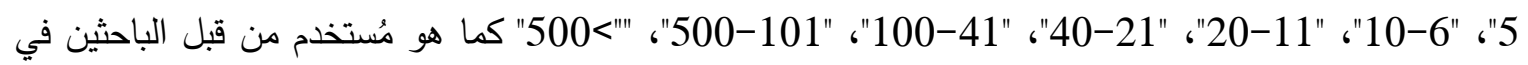

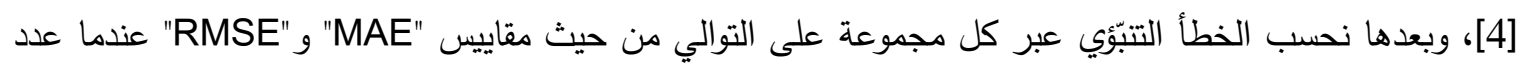

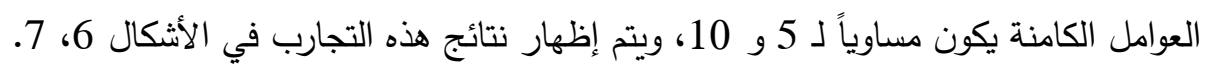

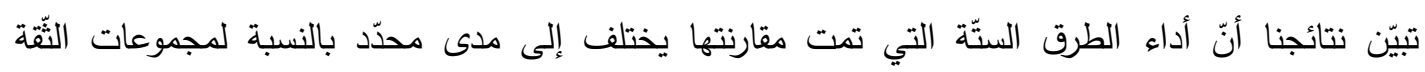

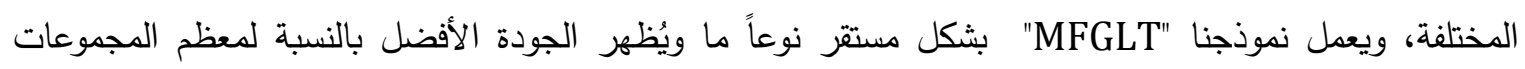

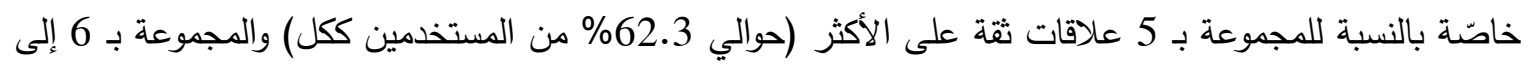

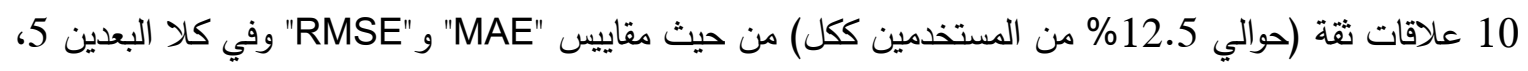

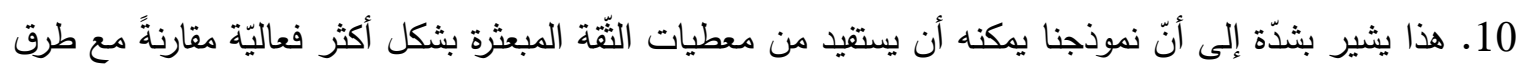
التصفية التعاونيّة الأخرى المعتمدة على التّّة. 
Journal of University of Babylon, Pure and Applied Sciences, Vol.(27), No.(1): 2019

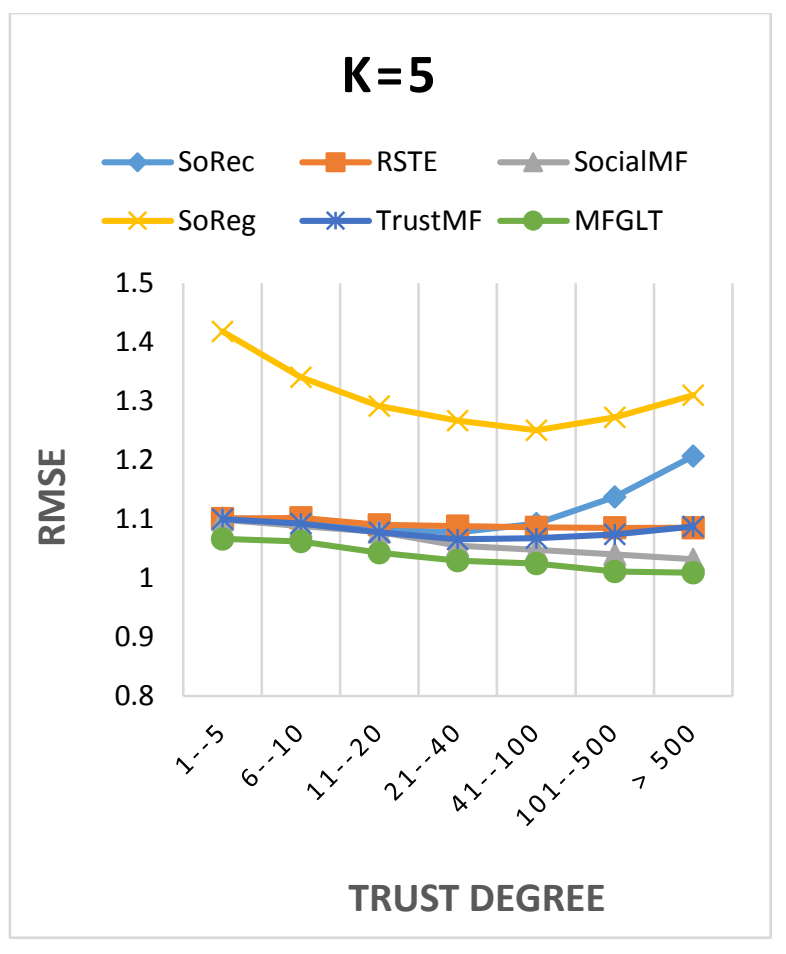

RMSE (b)

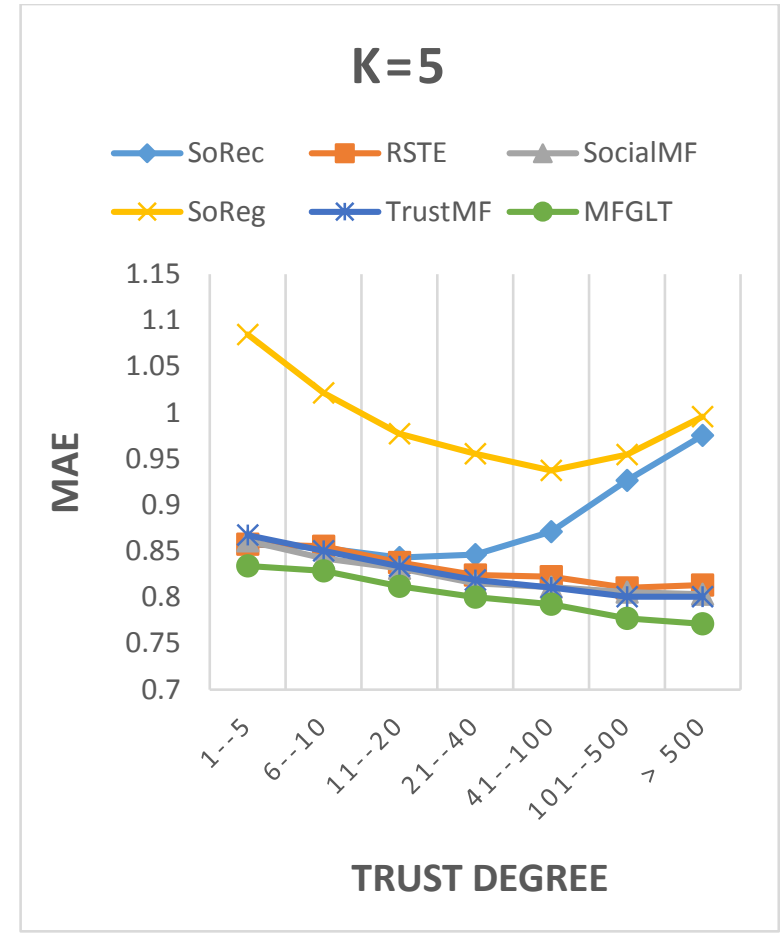

$\operatorname{MAE}(a)$

K = الثكل 6. الأداء التنبّؤي على المستخدمين بارجات مختلفة من علاقات الثّقة عندما

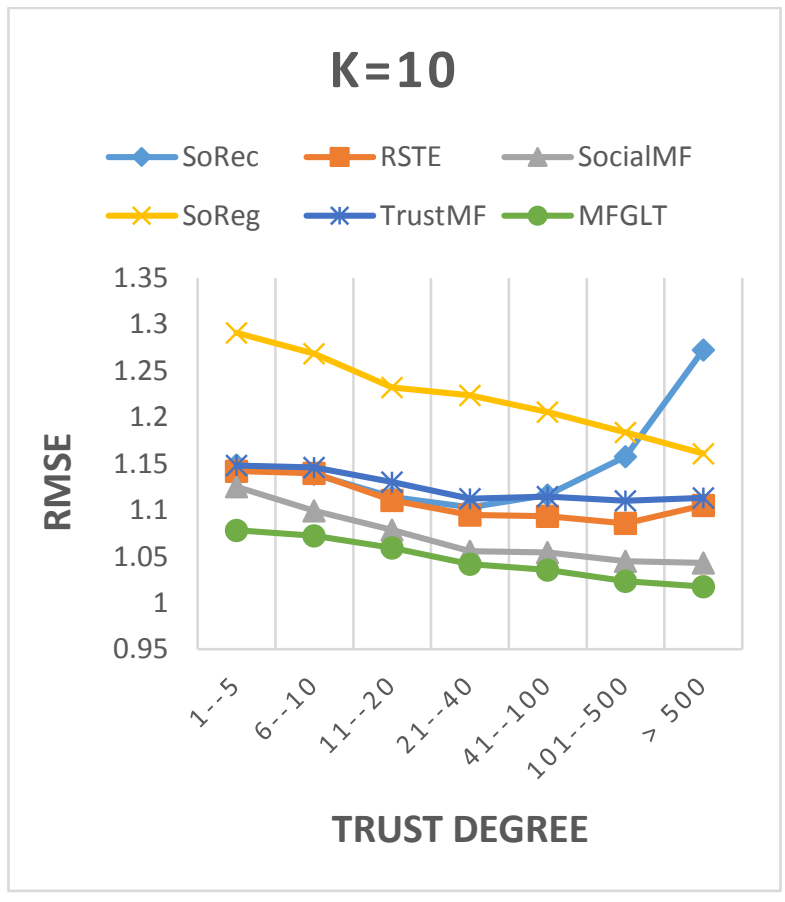

RMSE (b)

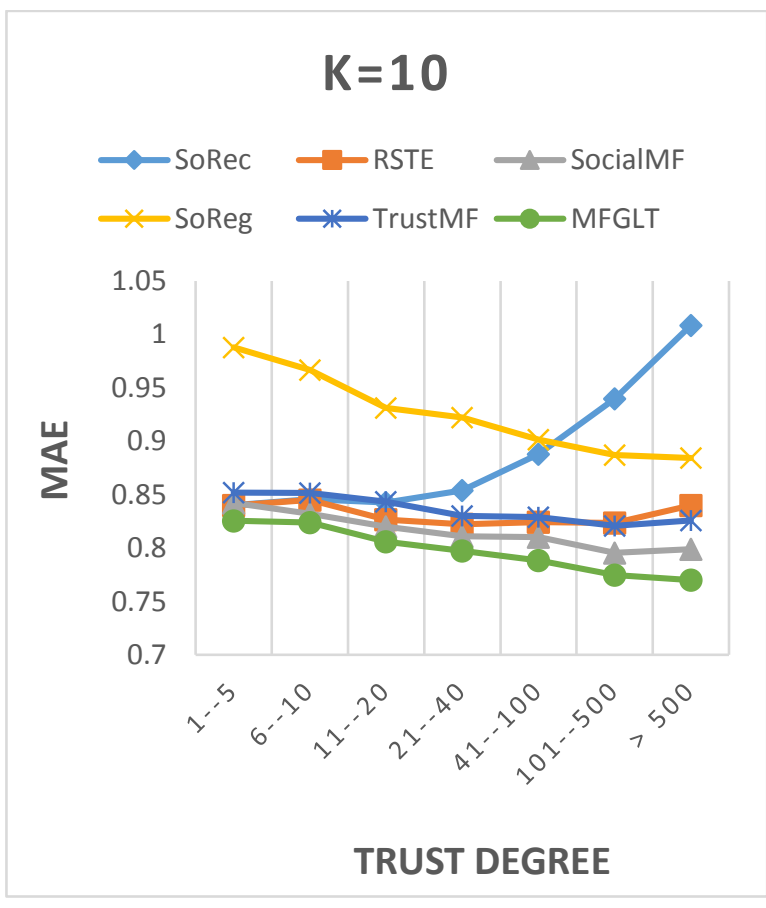

MAE (a)

K = الثكل 7. الأداء التنبؤي على المستخدمين بلرجات مختلفة من علاقات الثّّقة عندما 10 


\section{5. الاستنتاجات والتوصيات}

قدّمنا في هذا البحث مقترحنا من أجل تحسين دقةّ التوصيات في طريقة التصفية التعاونيّة المعتمدة على فلى النموذج بالاستفادة من علاقات التّّة بين المستخدمين، وذلك من خلال تقديم استراتيجيّة جديدة لصهر معطيات التقييم ومعطيات الثّقة للتعبير عن التأثير المتبادل للمستخدمين على آراء بعضهم البعض بشكل منطقي أكثر، وبالتالي التنبوّ

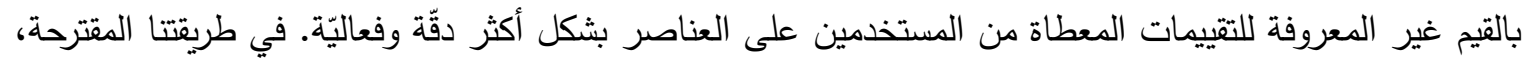
تمثّل المعلومات المحليّة تفضيلات الحالتين المختلفتين للمستخدم (كواثق وكموثوق به) والتي يتم تعلّمها عن طريق طئيق

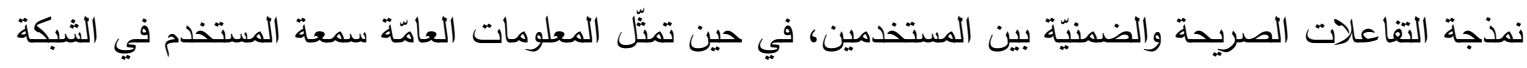
الاجتماعيّة ككل. بثكل أكثر تحديداً، قمنا بنمذجة التفاعلات الصريحة عن طريق تخمين التفضين تصنيلات الخاصّة بالواثقين والتفضيلات الخاصّة بالموثوقين بحيث تلائم التقييمات الصريحة وعلاقات الثّقة الصريحة، بينما قمنا باستغلال بنية الروابط المحليّة لشبكة الثّّة كحدود تتظيميّة لنمذجة الارتباط الضمنيّ للمستخدم مع المستخدمين

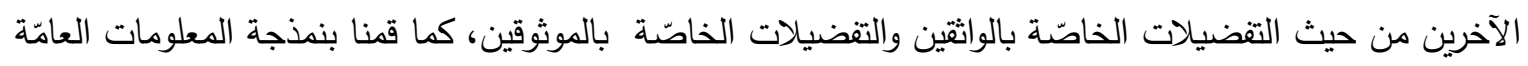
باستخدام أحد الخوارزميات الأكثر شعبيّة لحساب قيم السمعة للمستخدمين وهي "PageRank". في في النهاية قمنا بتشكيل نموذج موحّد يتضمن كلا النوعين من المعلومات.

تمّ إجراء تجارب على مجموعة معطيات حقيقيّة وهي "Epinions"

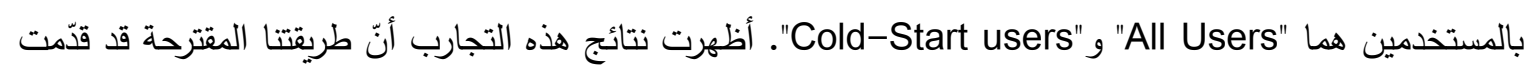

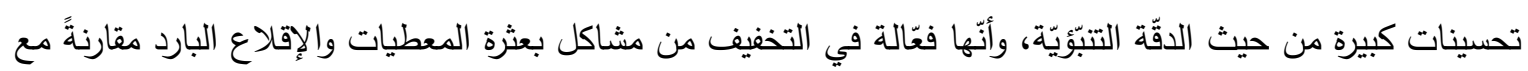

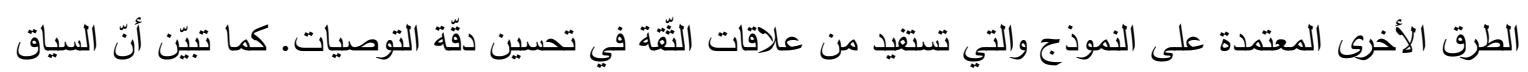

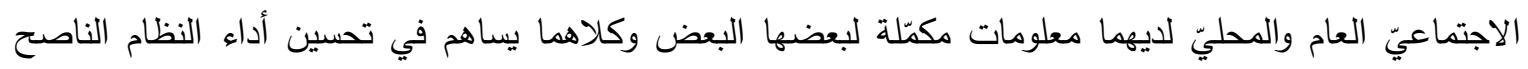

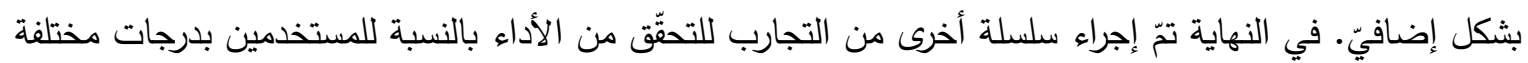
من علاقات الثّقة، والتي بيّنت أنّ نموذجنا المقترح يمكنه أن يستفيد من معطيات الثّّة المبعثرة بشكل أكثر فعاليّة مقارنةً مع طرق التصفية التعاونيّة الأخرى المعتمدة على الثّة.

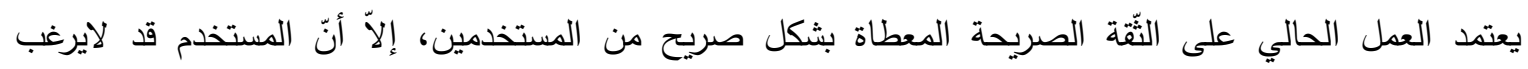
بمشاركة أو كثف هكذا معلومات بسبب مخاوف الخصوصية مثلاً، كما أنّ الكثير من مجموعات المعطيات المتاحة لاتحوي معلومات الثقة الصريحة، لذلك يمكن كعمل مستقبلي استتاج قيم الثقة الضمنيّة من سلوكيات المستخدم CONFLICT OF INTERESTS.

لتعزيز العموميّة لطريقتا المقترحة.

There are non-conflicts of interest.

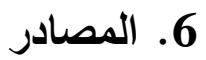

[1] X. Zhou, Y. Xu, Y. Li, A. Josang and C. Cox, "The state-of-the-art in personalized recommender systems for social networking", Artificial Intelligence Review, vol. 37, no. 2, pp. 119-132, 2011.

[2] G. Adomavicius and A. Tuzhilin, "Toward the next generation of recommender systems: a survey of the state-of-the-art and possible extensions", IEEE Transactions on Knowledge and Data Engineering, vol. 17, no. 6, pp. 734-749, 2005.

[3] Y. Koren, R. Bell and C. Volinsky, "Matrix Factorization Techniques for Recommender Systems", Computer, vol. 42, no. 8, pp. 30-37, 2009. 
[4] B. Yang, Y. Lei, J. Liu and W. Li, "Social Collaborative Filtering by Trust", IEEE Transactions on Pattern Analysis and Machine Intelligence, vol. 39, no. 8, pp. 1633-1647, 2017.

[5] H. Ma, H. Yang, M. Lyu and I. King, "SoRec: social recommendation using probabilistic matrix factorization", Proceeding of the 17th ACM conference on Information and knowledge mining - CIKM '08, pp. 931-940, 2008.

[6] H. Ma, I. King and M. Lyu, "Learning to recommend with social trust ensemble", Proceedings of the 32nd international ACM SIGIR conference on Research and development in information retrieval - SIGIR '09, pp. 203-210, 2009.

[7] M. Jamali and M. Ester, "A matrix factorization technique with trust propagation for recommendation in social networks", Proceedings of the fourth ACM conference on Recommender systems - RecSys '10, pp. 135-142, 2010.

[8] H. Ma, D. Zhou, C. Liu, M. Lyu and I. King, "Recommender systems with social regularization", Proceedings of the fourth ACM international conference on Web search and data mining - WSDM '11, pp. 287-296, 2011.

[9] P. Massa, "A survey of trust use and modeling in real online systems", Trust in Eservices: Technologies, Practices and Challenges, pp. 51-83, 2007.

[10] N. Agarwal, H. Liu, L. Tang and P. Yu, "Identifying the influential bloggers in a community", Proceedings of the international conference on Web search and web data mining - WSDM '08, pp. 207-218, 2008.

[11] J. Weng, E. Lim, J. Jiang and Q. He, "TwitterRank", Proceedings of the third ACM international conference on Web search and data mining - WSDM '10, pp. 261-270, 2010.

[12] J. Tang, X. Hu, H. Gao and H. Liu, "Exploiting local and global social context for recommendation", IJCAI, pp. 2712-2718, 2013.

[13] J. Rennie and N. Srebro, "Fast maximum margin matrix factorization for collaborative prediction", Proceedings of the 22nd international conference on Machine learning - ICML '05, pp. 713-719, 2005.

[14] Y. Koren, "Factorization meets the neighborhood", Proceeding of the 14th ACM SIGKDD international conference on Knowledge discovery and data mining - KDD 08, pp. 426-434, 2008.

[15] Y. Fang and L. Si, "Matrix co-factorization for recommendation with rich side information and implicit feedback", Proceedings of the 2nd International Workshop on Information Heterogeneity and Fusion in Recommender Systems - HetRec '11, pp. 65-69, 2011.

[16] A. Mnih and R. Salakhutdinov, "Probabilistic matrix factorization", Advances in neural information processing systems, pp. 1257-1264, 2008.

[17] J. Tang and H. Liu, "Trust in social media", Synthesis Lectures on Information Security, Privacy, \& Trust, vol. 10, no. 1, pp. 1-129, 2015.

[18] L. Page, S. Brin, R. Motwani and T. Winograd, "The PageRank citation ranking: Bringing order to the web", Stanford InfoLab, 1999.

[19] J. Kleinberg, "Authoritative sources in a hyperlinked environment", Journal of the $A C M$, vol. 46, no. 5, pp. 604-632, 1999.

[20] Y. Koren, "Factor in the neighbors: Scalable and accurate collaborative filtering", ACM Transactions on Knowledge Discovery from Data, vol. 4, no. 1, pp. 1-24, 2010 . 